\title{
Nonlinear Reduced-Order Observer-Based Predictive Control for Diving of an Autonomous Underwater Vehicle
}

\author{
Xuliang Yao, ${ }^{1}$ Guangyi Yang, ${ }^{1}$ and Yu Peng ${ }^{2}$ \\ ${ }^{1}$ College of Automation, Harbin Engineering University, Harbin 150001, China \\ ${ }^{2}$ Beijing Aerospace Automatic Control Institute, Beijing 100854, China \\ Correspondence should be addressed to Guangyi Yang; hahaygy@hrbeu.edu.cn
}

Received 15 October 2016; Accepted 14 December 2016; Published 3 January 2017

Academic Editor: Juan R. Torregrosa

Copyright (c) 2017 Xuliang Yao et al. This is an open access article distributed under the Creative Commons Attribution License, which permits unrestricted use, distribution, and reproduction in any medium, provided the original work is properly cited.

\begin{abstract}
The attitude control and depth tracking issue of autonomous underwater vehicle (AUV) are addressed in this paper. By introducing a nonsingular coordinate transformation, a novel nonlinear reduced-order observer (NROO) is presented to achieve an accurate estimation of AUV's state variables. A discrete-time model predictive control with nonlinear model online linearization (MPCNMOL) is applied to enhance the attitude control and depth tracking performance of AUV considering the wave disturbance near surface. In AUV longitudinal control simulation, the comparisons have been presented between NROO and full-order observer (FOO) and also between MPC-NMOL and traditional NMPC. Simulation results show the effectiveness of the proposed method.
\end{abstract}

\section{Introduction}

Autonomous underwater vehicle (AUV) is an important tool for ocean exploitation. Due to its strong autonomous ability, AUV can complete the mission given by human and has become widely used in military and scientific research [1].

Underactuated AUV is a kind of underwater vehicles whose actual control inputs are less than degrees of freedom (DOF) [2]. When AUV navigates near surface, diving control is one of the key research fields; a variety of methods have been proposed for AUV in vertical plane. Yan et el. [3] proposed backstepping technology and IFTSMC for surge velocity and depth control, respectively. However, the coupled interaction between the two controllers was not considered. An improved dynamic fuzzy SMC is established to enhance the depth tracking control performance in [4]. Adhami-Mirhosseini et al. [5] proposed a nonlinear dynamic controller combined with Fourier series expansion and pseudospectral ideas to achieve the depth tracking objective. In [6], an analytical SMC method is used to obtain the time optimal trajectory tracking, and the effectiveness of the proposed controller was verified via simulations. The fuzzy feedback linearization methods studied in [7] abandoned two general assumptions and used the nonlinear dynamics of depth motion directly. However, discrete-time model was not taken into consideration, and there is lack of simulation verification of the proposed algorithm. Hsu and Liu [8] investigated a steady-state depth errors problem caused by center of gravity change and considered the effects of gravity and buoyancy in AUV dynamics. By adding a switching PI controller in external-loop, the steady-state depth errors were eliminated finally. A static output feedback controller was studied in [9] to complete the diving task; however a linearized model was used.

Model predictive control can calculate a sequence of future control signals in advance, in order to track a given future reference. Moreover, predictive control allows the integration of system constraints in the controller design and minimizes a cost function defined over a prediction horizon, ensuring near-optimal performance of the system. The predictive control of AUV motion is one of the promising research areas in marine control systems field. In [10], visual servoing MPC is used to bring current-influenced AUV aperiodic algorithm-generated control loop closures. A cost function minimization method is used in [11] with MPC, called least squares, whose advantage is the real-time execution ability to optimize sawtooth paths for an underwater glider. A reduced dynamical model proposed in [12] is used to control AUV in vertical and horizontal planes, respectively. 
The MPC is formulated as two independent optimization parts. However, by using the same initial states for both parts of linearization and neglecting the weakly coupled states, the control optimization differs from the actual situation a lot. In [13], a sampling based MPC is proposed to generate the control sequence with constraints effectively. These methods have a good performance in the field of MPC use for AUV, but they do not deal with wave disturbances. The method in [14] used MPC to predict future disturbances and counteract the wave disturbances. A linear wave theory solver is employed by estimator to approximate the fluid dynamics, but constraints were not considered. NMPC is an extension of MPC by using nonlinear systems and constraints; applications of NMPC for AUV are less frequent. In [15], NMPC is used to design the kinematic loop in a hierarchical structure for AUV DP control.

Because of fewer actuators and sensors, there is an advantage for underactuated AUV to simplify the design and reduce costs. However, it brings highly coupled nonlinear models, which increases the difficulty of controller design. In general, some state variables of AUV are unmeasured due to a lack of sensors or sensor faults. So an observer-based controller becomes significant. A dual observer in [16], which combines a state and a perturbation observer, aims to solve the problem about being sensitive to external disturbance. Zhang et al. [17] considered the elevator angle constraints and proposed a controller based on MPC with artificial bee colony algorithm, and a classical linear state observer is used. In [18], a novel discrete-time proportional and integral observer is used to estimate the states, output, input, and disturbance together. Zhang et al. [19] proposed a new reduced-order observer with multiconstrained thoughts by using specific system decomposition. However, these results only use linear models.

In contrast, most practical systems are nonlinear and therefore nonlinear models are required. Kinsey et al. [20] proposed a nonlinear observer based on dynamic model of AUV, which is used to estimate the vehicle's velocity. However, in their model, the coupling terms are neglected and there was only one degree of freedom. Mahapatra et al. [21] investigated a nonlinear $H_{\infty}$ feedback control algorithm with a nonlinear state observer, but the error dynamics stability was not considered in observer design. Zhang et al. [22] extended an adaptive state observer to a class of nonlinear systems. However, due to the selected special Lyapunov matrix, there is a reduction of the accuracy of state estimation.

At the same time, MPC need state variables to achieve desired output tracking in the minimization of cost function, so the role of observer-based MPC has a prominent position in practical implementation. However, there is a situation that some states variables of underactuated AUV can be measured. So it is not necessary to estimate all the variables. Compared with FOO, reduced-order observer (ROO) has simplified structure and good estimation performance [23, 24], which can be designed to estimate only the unmeasurable states. Recently, ROO designs used in MPC are exploited in both linear [25, 26] and nonlinear systems [27-30]. However, few research results are reported on NROO-based MPC for AUV. So the motivation of this paper is the aforementioned problems and deficiencies, and the aim is to design a NROObased MPC with nonlinear AUV kinematics and dynamics model.

In this paper, a model predictive controller with nonlinear model online-linearization based on NROO is proposed to solve the attitude control and depth tracking issues. The design process is as follows. First, the nonlinear dynamics in AUV vertical plane are described and Euler approximation is used to discrete the proposed model. Second, the nonlinear reduced-order observer is designed according to the dynamics above, to estimate the unmeasured state variables of AUV. By solving a convex optimization problem and using LMI technique, the estimation performance is enhanced and the wave disturbance influence is restricted. Third, a model predictive control based on model onlinelinearization is presented, in which the controller design process is independent of the NROO and it is convenient for the following simulation. Finally, simulation results show the good robustness and efficiency of the proposed methods, which can be applied to AUV attitude control and depth tracking field.

The rest of the paper is organized as follows. Section 2 described the AUV vertical plane dynamics. NROO design is proposed in Section 3. Section 4 introduces the MPC-NMOL strategy. Section 5 gives the simulation results of AUV to verify the effectiveness of the proposed approach.

\section{AUV Vertical Plane Dynamics}

The vertical plane dynamic and kinematic model of AUV are described in this section, and the following assumptions are considered.

Assumption 1. (1) AUV navigates at a constant speed $u=U_{c}$.

(2) To analyze the diving control, sway, yaw, and roll motions can be ignored, and that means $y=0, v=0$, and $\phi=\psi=0$.

(3) Center of buoyancy stays constant with no change made to external hull, but the position of center of gravity is variable.

(4) The hydrodynamics drag terms whose order is higher than two are neglected.

(5) Pitch angle $\theta$ has small perturbations at zero.

Therefore, the dynamic and kinematic model of AUV can be expressed in the nonlinear forms as follows:

$$
\begin{aligned}
m\left[\dot{\omega}-u q-x_{g} \dot{q}\right] & =\Sigma Z_{\text {ext }}, \\
I_{y} \dot{q}+m\left[z_{g} \omega q-x_{g} \dot{\omega}+x_{g} u q\right] & =\Sigma M_{\text {ext }}, \\
\dot{z} & =-u \theta+\omega, \\
\dot{\theta} & =q,
\end{aligned}
$$

where

$$
\begin{aligned}
\Sigma Z_{\text {ext }}= & Z_{H S}+Z_{\omega|\omega|} \omega|\omega|+Z_{\dot{\omega}} \dot{\omega}+Z_{\dot{q}} \dot{q}+Z_{u q} u q \\
& +Z_{u \omega} u \omega+Z_{\delta} u^{2} \delta+Z_{\text {wave }} \\
\Sigma M_{\text {ext }}= & M_{H S}+M_{q|q|} q|q|+M_{\dot{\omega}} \dot{\omega}+M_{\dot{q}} \dot{q}+M_{u q} u q \\
& +M_{u \omega} u \omega+M_{\delta} u^{2} \delta+M_{\text {wave }},
\end{aligned}
$$




$$
\begin{aligned}
Z_{H S} & =(W-B) \cos \theta \\
M_{H S} & =\left(z_{b} B-z_{g} W\right) \theta+\left(x_{b} B-x_{g} W\right) .
\end{aligned}
$$

$I_{y}, m$ are the moment of inertia and the mass of AUV, respectively, $\left(x_{g}, y_{g}, z_{g}\right)$ and $\left(x_{b}, y_{b}, z_{b}\right)$ are the coordinates of AUV's center of gravity and buoyancy, $u, \omega$, and $q$ are the surge, heave, and pitch angle velocity in body-fixed frame, respectively, $z$ are depth in earth-fixed frame, $\delta$ is the elevator deflection angle, $Z_{\omega|\omega|} \omega|\omega|, Z_{\dot{\omega}}, Z_{\dot{q}}, Z_{u q}, Z_{u \omega}, Z_{\delta}$, $M_{q|q|} q|q|, M_{\dot{\omega}}, M_{\dot{q}}, M_{u q}, M_{u \omega}$, and $M_{\delta}$ are the hydrodynamics coefficients of AUV, and $Z_{\text {wave }}, M_{\text {wave }}$ are the wave force and moment.

The nonlinear model (1) can be simply expressed as the following system:

$$
\begin{aligned}
\dot{x}_{c}(t) & =A_{c} x_{c}(t)+g_{c}(t, x(t))+B_{c} u(t)+D_{c} w(t), \\
y(t) & =C x_{c}(t),
\end{aligned}
$$

where

$$
\begin{aligned}
& A_{c}=M_{1}^{-1}\left[\begin{array}{cccc}
n_{11} & n_{12} & 0 & 0 \\
n_{21} & n_{22} & 0 & n_{24} \\
1 & 0 & 0 & -u \\
0 & 1 & 0 & 0
\end{array}\right], \\
& B_{c}=M_{1}^{-1}\left[\begin{array}{c}
Z_{\delta} \\
M_{\delta} \\
0 \\
0
\end{array}\right], \\
& D_{c}=M_{1}^{-1}\left[\begin{array}{c}
I_{2 \times 2} \\
0_{2 \times 2}
\end{array}\right], \\
& g_{c}(t, x(t))
\end{aligned}
$$$$
=M_{1}^{-1}\left[\begin{array}{c}
Z_{\omega|\omega|} \omega|\omega|+(W-B) \\
-m z_{g} \omega q+M_{q|q|} q|q|-\left(x_{g} W-x_{b} B\right) \\
0 \\
0
\end{array}\right],
$$$$
M_{1}=\left[\begin{array}{cccc}
m_{11} & m_{12} & 0 & 0 \\
m_{21} & m_{22} & 0 & 0 \\
0 & 0 & 1 & 0 \\
0 & 0 & 0 & 1
\end{array}\right]
$$$$
C=\left[\begin{array}{llll}
0 & 0 & 1 & 0 \\
0 & 0 & 0 & 1
\end{array}\right] \text {, }
$$$$
m_{11}=m-Z_{\dot{\omega}} \text {, }
$$$$
m_{12}=-Z_{\dot{q}}-m x_{g}
$$$$
m_{21}=-m x_{g}-M_{\dot{\omega}} \text {, }
$$$$
m_{22}=I_{y}-M_{\dot{q}}
$$$$
n_{11}=Z_{u \omega} U_{c} \text {, }
$$

$$
\begin{aligned}
& n_{12}=\left(Z_{u q}+m U_{c}\right), \\
& n_{21}=M_{u \omega} U_{c}, \\
& n_{22}=\left(M_{u q}-m x_{g} U_{c}\right), \\
& n_{24}=-\left(z_{g} W-z_{b} B\right) .
\end{aligned}
$$

Due to the digital controller implemented by using zero-order hold, a discrete-time vector-form nonlinear state equation is obtained by Euler approximation [31].

$$
\begin{aligned}
x(k+1) & =A x(k)+g(k, x(k))+B u(k)+D w(k), \\
y(k) & =C x(k),
\end{aligned}
$$

where $x(k)=\left[x_{1}, x_{2}, x_{3}, x_{4}\right]^{T} \in R^{n}, x_{1}=\omega, x_{2}=q, x_{3}=z$, $x_{4}=\theta, T_{s}$ is the sampling period, $A=\left(T_{s} A_{c}+I\right), B=T_{s} B_{c}$, $D=T_{s} D_{c}, w(k)=\left[Z_{\text {wave }} \quad M_{\text {wave }}\right] \in R^{d}, u(k) \in R^{m}$ is the input $\delta, g(k, x(k))=T_{s} g_{c}(t, x(t))$ is a nonlinear-term vector, and $y(k) \in R^{p}$ denotes the output vector. In this paper, $n=4$, $d=2, m=1, p=2, C$ is a full-rank matrix, and $\operatorname{rank}(C)=2$, and we assume that pair $(A, C)$ is observable.

Remark 2. In this paper, the disturbance due to surface waves is considered as the main disturbance type and written as $w(k)$. Sea state is used to classify the sea conditions and expressed by wind speed and wave height. Wave height is affected by wind, and the wave profile is affected by water depth. For surface waves modelling, we assume the long crested and unidirectional sea state. The superposition principle is used to complete an irregular long crested wave sea state simulation. The single parameter Pierson-Moskowitz spectrum [32] is used in this model and its spectrum density is defined as follows:

$$
S(\omega)=\frac{8.1 \times 10^{-3} g^{2}}{\bar{\omega}^{5}} \exp \left[\frac{-3.11}{H_{s}^{2} \bar{\omega}^{4}}\right],
$$

where $H_{s}$ is the significant wave height (m) and $\bar{\omega}$ is wave frequency. Assuming the AUV body is small enough compared with the incoming wave and the wave speed is large enough in relation to the hull diameter $D_{0}$, the transient force and moment are found by using Morison's equation [33] and integration along the length of the hull $L_{0}$ at each step is obtained below:

$$
\begin{gathered}
Z_{\text {wave }}=\int_{L_{0}}\left(C_{d} \frac{\rho D_{0}}{2}\left(\omega_{\omega}-\omega\right)^{2}\right. \\
\left.+C_{m} \frac{\rho \pi D_{0}^{2}}{4}\left(\dot{\omega}_{\omega}-\dot{\omega}\right)^{2}\right) d x, \\
M_{\text {wave }}=\int_{L_{0}}\left(C_{d} \frac{\rho D_{0}}{2}\left(\omega_{\omega}-\omega\right)^{2}\right. \\
\left.+C_{m} \frac{\rho \pi D_{0}^{2}}{4}\left(\dot{\omega}_{\omega}-\dot{\omega}\right)^{2}\right) x d x,
\end{gathered}
$$




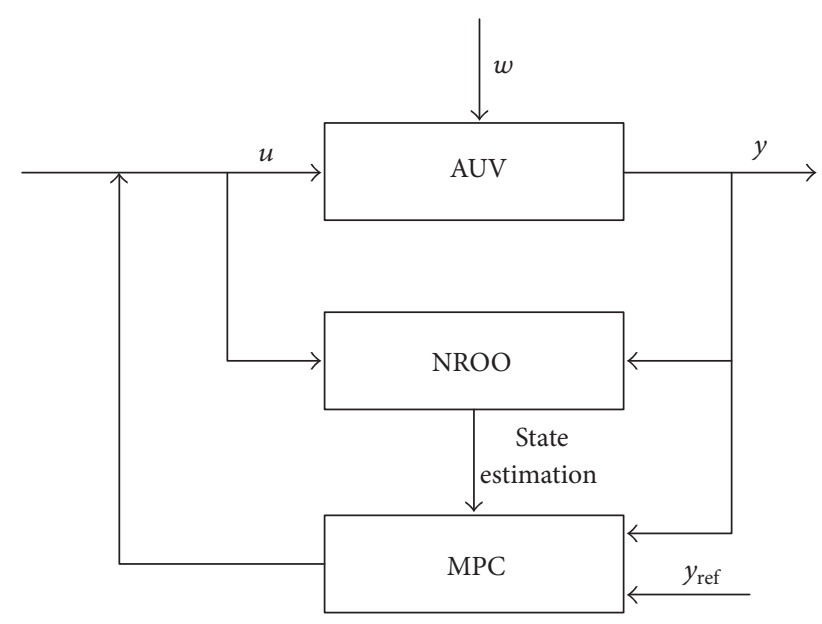

FIGURE 1: Block diagram of the proposed system.

where $\rho$ is fluid density, $C_{d}$ is the drag coefficient, and $C_{m}$ is the added mass coefficient.

In (7),

$$
\begin{aligned}
& \omega_{\omega}=\sum_{i=1}^{N} \omega_{e i} \alpha_{i} \exp \left(-k_{i} z\right) \sin \left(\omega_{e i} t+\theta_{i}\right), \\
& \dot{\omega}_{\omega}=-\sum_{i=1}^{N} \omega_{e i}{ }^{2} \alpha_{i} \exp \left(-k_{i} z\right) \cos \left(\omega_{e i} t+\theta_{i}\right),
\end{aligned}
$$

where $\omega_{e i}$ is encountering frequency, $\alpha_{i}=\sqrt{2 S\left(\bar{\omega}_{i}\right) d \bar{\omega}}, N$ is the equal interval frequency waveband and chosen in this article to discretize the wave spectrum, $k_{i}$ is wave number, and $\theta_{i}$ is random phase shift $\left(0<\theta_{i}<2 \pi\right)$ in relation to each frequency.

Assumption 3. $g(k, x(k))$ is a Lipschitz function [34] vector:

$$
\left\|g\left(k, \bar{x}_{1}\right)-g\left(k, \bar{x}_{2}\right)\right\|_{2} \leq L_{g}\left\|\bar{x}_{1}-\bar{x}_{2}\right\|_{2}
$$

which is assumed to satisfy the following conditions:

(1) The effect of $Z_{H S}$ is approximated to zero.

(2) $x_{g} \approx x_{b}=0$,

where $L_{g}>0$ is a Lipschitz constant and $\|\cdot\|_{2}$ denotes the Euclidean norm. Moreover, $g(0, k)=0$.

Remark 4. This paper proposed MPC-NMOL design method based on NROO for a nonlinear Lipschitz model of AUV. The NROO is independent of the MPC-NMOL, which is constructed to guarantee the stability and performance of AUV diving motion. Figure 1 shows the structure of the proposed system.

\section{Nonlinear Reduced-Order Observer Design}

3.1. NROO Algorithm Design. Consider that $C$ is full-row rank, and there always exists a matrix $C^{\perp} \in R^{(n-p) \times n}$ such that
$\left[\begin{array}{c}C^{\perp} \\ C\end{array}\right] \in R^{n \times n}$ is nonsingular $\left(C^{\perp}\right.$ can be given as an orthogonal basis of the null-space of $C$ ). Then under the nonsingular coordinate transformation $z(k)=T x(k)$, in which $T=\left[\begin{array}{c}C^{\perp} \\ C\end{array}\right]$ is used, the dynamics (5) can be expressed into the form

$$
\begin{aligned}
{\left[\begin{array}{c}
z_{1}(k+1) \\
z_{2}(k+1)
\end{array}\right]=} & {\left[\begin{array}{ll}
A_{11} & A_{12} \\
A_{21} & A_{22}
\end{array}\right]\left[\begin{array}{l}
z_{1}(k) \\
z_{2}(k)
\end{array}\right] } \\
& +\left[\begin{array}{c}
C^{\perp} \\
C
\end{array}\right] g\left(k, T^{-1} z(k)\right) \\
& +\left[\begin{array}{c}
B_{1} \\
B_{2}
\end{array}\right] u(k)+\left[\begin{array}{c}
D_{1} \\
D_{2}
\end{array}\right] w(k), \\
y(k)= & {\left[\begin{array}{l}
0_{p \times(n-p)} \\
I_{p}
\end{array}\right]\left[\begin{array}{l}
z_{1}(k) \\
z_{2}(k)
\end{array}\right], }
\end{aligned}
$$

where $z_{1}(k) \in R^{n-p}$ and $z_{2}(k) \in R^{p}$ are new state vectors, $I_{p}$ is identity matrix whose dimension is $p \times p$, and

$$
\begin{aligned}
& \text { TAT }^{-1}=\left[\begin{array}{ll}
A_{11} & A_{12} \\
A_{21} & A_{22}
\end{array}\right], \\
& T B=\left[\begin{array}{l}
B_{1} \\
B_{2}
\end{array}\right], \\
& T D=\left[\begin{array}{l}
D_{1} \\
D_{2}
\end{array}\right], \\
& C T^{-1}=\left[\begin{array}{ll}
0_{p \times(n-p)} & I_{p}
\end{array}\right], \\
& z(k)=T x(k)=\left[\begin{array}{l}
z_{1}(k) \\
z_{2}(k)
\end{array}\right],
\end{aligned}
$$

and then

$$
\begin{aligned}
z_{2}(k)= & y(k), \\
z_{1}(k+1)= & A_{11} z_{1}(k)+A_{12} y(k) \\
& +C^{\perp} g\left(k, T^{-1} z(k)\right)+B_{1} u(k) \\
& +D_{1} w(k), \\
y(k+1)= & A_{21} z_{1}(k)+A_{22} y(k)+C g\left(k, T^{-1} z(k)\right) \\
& +B_{2} u(k)+D_{2} w(k) .
\end{aligned}
$$

By using the hypothesis input $\mu(k)$ and output $\rho(k)$ :

$$
\begin{aligned}
& \mu(k)=A_{12} y(k)+B_{1} u(k), \\
& \rho(k)=y(k+1)-A_{22} y(k)-B_{2} u(k)
\end{aligned}
$$


we get

$$
\begin{aligned}
z_{1}(k+1)= & A_{11} z_{1}(k)+C^{\perp} g\left(k, T^{-1} z(k)\right)+\mu(k) \\
& +D_{1} w(k), \\
\rho(k)= & A_{21} z_{1}(k)+C g\left(k, T^{-1} z(k)\right)+D_{2} w(k) .
\end{aligned}
$$

From the dynamics (14), NROO is constructed as

$$
\begin{aligned}
\widehat{z}_{1}(k+1)= & A_{11} \widehat{z}_{1}(k)+C^{\perp} g\left(k, T^{-1} \widehat{z}(k)\right)+\mu(k) \\
& -G(\widehat{\rho}(k)-\rho(k)), \\
\hat{\rho}(k)= & A_{21} \widehat{z}_{1}(k)+C g\left(k, T^{-1} \widehat{z}(k)\right),
\end{aligned}
$$

where $\widehat{z}_{1}(k) \in R^{n-p}$ is the observer state of the reducedorder system (14), $\hat{\rho}(k) \in R^{p}$ is the output of reduced-order observer, and $G \in R^{(n-p) \times p}$ is the observer gain matrix.

Denote

$$
\begin{aligned}
e(k) & =\widehat{z}_{1}(k)-z_{1}(k), \\
G(k) & =g\left(k, T^{-1} \widehat{z}(k)\right)-g\left(k, T^{-1} z(k)\right)
\end{aligned}
$$

and then, from (14)-(16), the state estimation error dynamics are described by the following:

$$
\begin{aligned}
e(k+1)= & \left(A_{11}-G A_{21}\right) e(k)+\left(C^{\perp}-G C\right) G(k) \\
& +\left(G D_{2}-D_{1}\right) w(k) .
\end{aligned}
$$

Equation (18) is rewritten as the following form:

$$
\begin{aligned}
e(k+1)= & \left(\bar{A}_{1}-G \bar{A}_{2}\right) e(k)+\left(G C_{2}-C_{1}\right) G(k) \\
& +\left(G D_{2}-D_{1}\right) w(k)
\end{aligned}
$$

where $\bar{A}_{1}=A_{11}, \bar{A}_{2}=A_{21}, C_{1}=-C^{\perp}$, and $C_{2}=-C$.

Theorem 5 gives a constrained NROO design, whose state estimation performance is specified via a $H_{\infty}$ performance index.

Theorem 5. Let a prescribed $H_{\infty}$ performance level $\gamma_{1}>0$, and if there exist a symmetric positive definite matrix $\bar{P} \in$ $R^{(n-p) \times(n-p)}$, a matrix $\bar{Y} \in R^{(n-p) \times p}$, and a $\varepsilon_{1}>0$ such that the following condition holds:

$$
\left[\begin{array}{ccccc}
-\bar{P} & \bar{P} \bar{A}_{1}-\bar{Y} \bar{A}_{2} & \bar{Y} C_{2}-\bar{P} C_{1} & \bar{Y} D_{2}-\bar{P} D_{1} & 0 \\
* & -\bar{P}+\varepsilon_{1} L_{g}^{2} I_{n-p} & 0 & 0 & I_{n-p} \\
* & * & -\varepsilon_{1} \lambda_{\min }\left(T^{T} T\right) I_{n} & 0 & 0 \\
* & * & * & -\gamma_{1} I_{d} & 0 \\
* & * & * & * & -\gamma_{1} I_{n-p}
\end{array}\right] \leq 0
$$

where $\bar{Y}=\bar{P} G$ and $*$ denotes the symmetric elements in a matrix, then the error dynamics (18) satisfy $H_{\infty}$ performance index $\|e(k)\|_{2} \leq \gamma_{1}\|w(k)\|_{2}$, and the NROO gain matrix can be obtained by $G=\bar{P}^{-1} \bar{Y}$.

Proof. Choose the following Lyapunov function:

$$
V(k)=e^{T}(k) \bar{P} e(k) \text {. }
$$

According to error dynamics (18) and (21), the difference $\Delta V(k)$ is

$$
\begin{aligned}
\Delta V(k) & =V(k+1)-V(k) \\
= & e^{T}(k+1) \bar{P} e(k+1)-e^{T}(k) \bar{P} e(k) \\
= & e^{T}(k)\left(\bar{A}_{1}-G \bar{A}_{2}\right)^{T} \bar{P}\left(\bar{A}_{1}-G \bar{A}_{2}\right) e(k) \\
& +G^{T}(k)\left(G C_{2}-C_{1}\right)^{T} \bar{P}\left(G C_{2}-C_{1}\right) G(k) \\
& +w^{T}(k)\left(G D_{2}-D_{1}\right)^{T} \bar{P}\left(G D_{2}-D_{1}\right) w(k) \\
& +2 e^{T}(k)\left(\bar{A}_{1}-G \bar{A}_{2}\right)^{T} \bar{P}\left(G C_{2}-C_{1}\right) G(k) \\
& +2 e^{T}(k)\left(\bar{A}_{1}-G \bar{A}_{2}\right)^{T} \bar{P}\left(G D_{2}-D_{1}\right) w(k) \\
& +2 G^{T}(k)\left(G C_{2}-C_{1}\right)^{T} \bar{P}\left(G D_{2}-D_{1}\right) w(k) \\
& -e^{T}(k) \bar{P} e(k) .
\end{aligned}
$$

Since $y(k)$ is measurable, $\widehat{z}_{2}(k)$ can be substituted by $y(k)$, and the observer state $\hat{x}(k)$ can be written as

$$
\widehat{x}(k)=T^{-1}\left[\begin{array}{c}
\widehat{z}_{1}(k) \\
y(k)
\end{array}\right] .
$$

One gets

$$
\begin{aligned}
\hat{x}(k)-x(k) & =T^{-1}\left[\begin{array}{c}
\widehat{z}_{1}(k) \\
y(k)
\end{array}\right]-T^{-1}\left[\begin{array}{c}
z_{1}(k) \\
y(k)
\end{array}\right] \\
& =T^{-1}\left[\begin{array}{c}
e(k) \\
0
\end{array}\right] .
\end{aligned}
$$

Since $G(k)=g\left(k, T^{-1} \widehat{z}(k)\right)-g\left(k, T^{-1} z(k)\right)$ and $g(k, x(k))$ satisfies the Lipschitz condition

$$
\|G(k)\|_{2} \leq L_{g}\|\hat{x}(k)-x(k)\|_{2}
$$

for a positive scalar $\varepsilon_{1}$ we have

$$
\begin{gathered}
\varepsilon_{1} G^{T}(k) G(k) \leq \varepsilon_{1} L_{g}^{2}(\widehat{x}(k)-x(k))^{T}(\widehat{x}(k)-x(k)) \\
=\varepsilon_{1} L_{g}^{2}\left[\begin{array}{ll}
e^{T}(k) & 0
\end{array}\right]\left(T^{-1}\right)^{T} T^{-1}\left[\begin{array}{c}
e(k) \\
0
\end{array}\right] .
\end{gathered}
$$


The above inequality is multiplied by $\lambda_{\min }\left(T^{T} T\right)$ on both sides, and we have

$$
\begin{aligned}
& \varepsilon_{1} \lambda_{\min }\left(T^{T} T\right) G^{T}(k) G(k) \\
& \leq \varepsilon_{1} L_{g}^{2} \lambda_{\min }\left(T^{T} T\right)\left[\begin{array}{ll}
e^{T}(k) & 0
\end{array}\right]\left(T^{-1}\right)^{T} T^{-1}\left[\begin{array}{c}
e(k) \\
0
\end{array}\right] \\
& \leq \varepsilon_{1} L_{g}^{2}\left[e^{T}(k) 0\right]\left(T^{T}\right)^{-1}\left(T^{T} T\right) T^{-1}\left[\begin{array}{c}
e(k) \\
0
\end{array}\right] \\
& =\varepsilon_{1} L_{g}^{2}\left[e^{T}(k) 0\right]\left[\begin{array}{c}
e(k) \\
0
\end{array}\right]=\varepsilon_{1} L_{g}^{2} e^{T}(k) e(k)
\end{aligned}
$$

which is

$$
\varepsilon_{1} L_{g}^{2} e^{T}(k) e(k)-\varepsilon_{1} \lambda_{\min }\left(T^{T} T\right) G^{T}(k) G(k) \geq 0,
$$

where $\lambda_{\min }(\cdot)$ is the smallest eigenvalue matrix.

We define

$$
J_{1}=\sum_{k=0}^{K}\left[\frac{1}{\gamma_{1}} e^{T}(k) e(k)-\gamma_{1} w^{T}(k) w(k)\right] .
$$

Under zero initial conditions, one gets

$$
J_{1} \leq \sum_{k=0}^{K}\left[\frac{1}{\gamma_{1}} e^{T}(k) e(k)-\gamma_{1} w^{T}(k) w(k)+\Delta V(k)\right]
$$

Substituting (22) and (28) into (30), it follows that

$$
\begin{aligned}
J_{1} & \leq \sum_{k=0}^{K}\left[\frac{1}{\gamma_{1}} e^{T}(k) e(k)-\gamma_{1} w^{T}(k) w(k)\right. \\
& +\varepsilon_{1} L_{g}^{2} e^{T}(k) e(k)-\varepsilon_{1} \lambda_{\min }\left(T^{T} T\right) G^{T}(k) G(k) \\
& +\Delta V(k)]=\sum_{k=0}^{K}\left[e^{T}(k) G^{T}(k) w^{T}(k)\right]
\end{aligned}
$$

$$
\cdot \Omega\left[\begin{array}{c}
e(k) \\
G(k) \\
w(k)
\end{array}\right],
$$

where

$$
\begin{aligned}
\Omega & =\left[\begin{array}{ccc}
c_{11} & \left(\bar{A}_{1}-G \bar{A}_{2}\right)^{T} \bar{P}\left(G C_{2}-C_{1}\right) & \left(\bar{A}_{1}-G \bar{A}_{2}\right)^{T} \bar{P}\left(G D_{2}-D_{1}\right) \\
* & c_{22} & \left(G C_{2}-C_{1}\right)^{T} \bar{P}\left(G D_{2}-D_{1}\right) \\
* & * & c_{33}
\end{array}\right], \\
c_{11} & =\left(\bar{A}_{1}-G \bar{A}_{2}\right)^{T} \bar{P}\left(\bar{A}_{1}-G \bar{A}_{2}\right)-\bar{P}+\varepsilon_{1} L_{g}^{2}+\frac{1}{\gamma_{1}} I_{n-p}, \\
c_{22} & =\left(G C_{2}-C_{1}\right)^{T} \bar{P}\left(G C_{2}-C_{1}\right)-\varepsilon_{1} \lambda_{\min }\left(T^{T} T\right) I_{n}, \\
c_{33} & =\left(G D_{2}-D_{1}\right)^{T} \bar{P}\left(G D_{2}-D_{1}\right)-\gamma_{1} I_{d} .
\end{aligned}
$$

By using the Schur complement lemma, $\Omega \leq 0$ is equivalent to

$$
\left[\begin{array}{cccc}
c_{11}-\frac{1}{\gamma_{1}} I_{n-p} & \left(\bar{A}_{1}-G \bar{A}_{2}\right)^{T} \bar{P}\left(G C_{2}-C_{1}\right) & \left(\bar{A}_{1}-G \bar{A}_{2}\right)^{T} \bar{P}\left(G D_{2}-D_{1}\right) & I_{n-p} \\
* & c_{22} & \left(G C_{2}-C_{1}\right)^{T} \bar{P}\left(G D_{2}-D_{1}\right) & 0 \\
* & * & c_{33} & 0 \\
* & * & * & -\gamma_{1} I_{n-p}
\end{array}\right] \leq 0 .
$$

Furthermore, (33) can be rewritten as

$$
\begin{aligned}
& {\left[\begin{array}{c}
\left(\bar{A}_{1}-G \bar{A}_{2}\right)^{T} \bar{P} \\
\left(G C_{2}-C_{1}\right)^{T} \bar{P} \\
\left(G D_{2}-D_{1}\right)^{T} \bar{P} \\
0
\end{array}\right] \bar{P}^{-1}\left[\bar{P}\left(\bar{A}_{1}-G \bar{A}_{2}\right) \bar{P}\left(G C_{2}-C_{1}\right)\right.} \\
& \left.\quad \bar{P}\left(G D_{2}-D_{1}\right) 0\right]
\end{aligned}
$$

$$
+\left[\begin{array}{cccc}
-\bar{P}+\varepsilon_{1} L_{g}^{2} & 0 & 0 & I_{n-p} \\
* & -\varepsilon_{1} \lambda_{\min }\left(T^{T} T\right) I_{n} & 0 & 0 \\
* & * & -\gamma_{1} I_{d} & 0 \\
* & * & * & -\gamma_{1} I_{n-p}
\end{array}\right]
$$$$
\leq 0 \text {. }
$$ 
Let us use Schur complement lemma again; finally, we obtain (20), which guarantees $\|e(k)\|_{2} \leq \gamma_{1}\|w(k)\|_{2}$. So if (20) holds, then (19) is exponentially stable with a $H_{\infty}$ performance index $\|e(k)\|_{2} \leq \gamma_{1}\|w(k)\|_{2}$.

Remark 6. We use the measurable output $y(k)=z_{2}(k)$ and the inequality $\lambda_{\text {min }}(P) a^{T} a \leq a^{T} P a$ for any matrix $P>0$ and real vector $a$, to make the Lipschitz condition transform into $\varepsilon_{1} \lambda_{\min }\left(T^{T} T\right) G^{T}(k) G(k) \leq \varepsilon_{1} L_{g}^{2} e^{T}(k) e(k)$, which guarantees the diagonal element $(3,3)$ is nonzero and negative and helps to form constraint (20).

Remark 7. In Theorem 5, the LMI constraint (20) estimates the states by suppressing the influence of the disturbance term $w(k)$ on $e(k)$. By solving the convex optimization problem, the level $\gamma_{1}$ can be obtained, in order to minimize $e(k)$. Apparently, the NROO order $n-p$ is less than the FOO [35] order $n$, and the transfer function from $w(k)$ to $e(k)$ is simplified. Therefore, NROO has the advantages of simple construction and better performance, which is compared to FOO.

3.2. NROO Existence Condition. On one hand, for the existence of NROO, the necessity of condition $\operatorname{rank}(C D)=$ $\operatorname{rank}(D)$ has been proved in [36]. It is called observability with unknown input, which means the necessity of condition can guarantee the observability of systems with unknown disturbances $w(k)$.

On the other hand, for the existence of FOO, the necessity of condition requires the pair $(A, C)$ to be observable [35]; that means

$$
\begin{gathered}
\operatorname{rank}\left(\begin{array}{cc}
\lambda I_{n-p}-A_{11} & -A_{12} \\
-A_{21} & \lambda I_{p}-A_{22} \\
0_{p \times(n-p)} & I_{p}
\end{array}\right) \\
=\operatorname{rank}\left(\begin{array}{c}
\lambda I_{n-p}-A_{11} \\
A_{21}
\end{array}\right)+p=n .
\end{gathered}
$$

It is not difficult to prove that the formula above is equivalent to the necessity of condition in NROO, because the nonsingular coordinate transformation which will be introduced in next section cannot transform the observability of the system, which means the NROO has the same range of application as FOO in this paper.

3.3. State Estimation. The NROO gain matrix $G$ has been derived in the previous section. However, hypothesis output $\rho(k)$ includes future output $y(k+1)$ which is not available in practical implementation. In this section, a novel expression will be introduced for state estimation.

Substituting (16) and (23) into (15), one gets

$$
\begin{aligned}
\widehat{z}_{1}(k+1)= & \left(A_{11}-G A_{21}\right) \widehat{z}_{1}(k) \\
& +\left(C^{\perp}-G C\right) g\left(k, T^{-1}\left[\begin{array}{c}
\widehat{z}_{1}(k) \\
y(k)
\end{array}\right]\right) \\
& +I_{n-p} \mu(k)+G \rho(k) \\
= & \left(\bar{A}_{1}-G \bar{A}_{2}\right) \widehat{z}_{1}(k)
\end{aligned}
$$

$$
\begin{aligned}
& +\left(G C_{2}-C_{1}\right) g\left(k, T^{-1}\left[\begin{array}{c}
\widehat{z}_{1}(k) \\
y(k)
\end{array}\right]\right) \\
& +I_{n-p} \mu(k)+G \rho(k) .
\end{aligned}
$$

Then, substituting $\mu(k)$ and $\rho(k)$ into (36) yields

$$
\begin{aligned}
\widehat{z}_{1}(k+1)= & \left(\bar{A}_{1}-G \bar{A}_{2}\right) \widehat{z}_{1}(k) \\
& +\left(G C_{2}-C_{1}\right) g\left(k, T^{-1}\left[\begin{array}{c}
\widehat{z}_{1}(k) \\
y(k)
\end{array}\right]\right) \\
& +\left(I_{n-p} A_{12}-G A_{22}\right) y(k)+G y(k+1) \\
& +\left(I_{n-p} B_{1}-G B_{2}\right) u(k) .
\end{aligned}
$$

We denote $\chi(k)=\widehat{z}_{1}(k)-G y(k)$ in order to eliminate $y(k+1)$ as follows:

$$
\begin{aligned}
\chi(k+1) & \\
= & \left(\bar{A}_{1}-G \bar{A}_{2}\right)(\chi(k)+G y(k)) \\
& +\left(G C_{2}-C_{1}\right) g\left(k, T^{-1}\left[\begin{array}{r}
\widehat{z}_{1}(k) \\
y(k)
\end{array}\right]\right) \\
& +\left(I_{n-p} A_{12}-G A_{22}\right) y(k) \\
& +\left(I_{n-p} B_{1}-G B_{2}\right) u(k) \\
= & \left(\bar{A}_{1}-G \bar{A}_{2}\right) \chi(k) \\
& +\left(G C_{2}-C_{1}\right) g\left(k, T^{-1}\left[\begin{array}{r}
\bar{z}_{1}(k) \\
y(k)
\end{array}\right]\right) \\
& +\left(\left(\bar{A}_{1}-G \bar{A}_{2}\right)+\left(I_{n-p} A_{12}-G A_{22}\right)\right) y(k) \\
& +\left(I_{n-p} B_{1}-G B_{2}\right) u(k) .
\end{aligned}
$$

Finally, the state estimator is

$$
\widehat{x}(k)=T^{-1}\left[\begin{array}{c}
\chi(k)+G y(k) \\
y(k)
\end{array}\right] .
$$

\section{Model Predictive Control with Nonlinear Model Online Linearization}

4.1. Nonlinear Predictive Model Online Linearization. In this section, MPC-NMOL was proposed to deal with the nonlinear state equation (5). Due to the time-consuming and computational complex problem, nonlinear optimization is converted into a quadratic optimization, by considering an online linearized method. Using gain scheduling technique [12], the online linearized dynamics at current sampling instance are given as

$$
\begin{aligned}
x(k+1) & =(A+\bar{g}(k, x)) x(k)+B u(k)+D w(k), \\
y(k) & =C x(k),
\end{aligned}
$$


where the nonlinear-term $\bar{g}(k, x)$ is linearized from $g(k, x(k))$, and one gets

$$
\bar{g}(x, k)=\left.\frac{\partial g(k, x(k))}{\partial x(k)}\right|_{x(k)=\widehat{x}(k)}
$$

where the current operating state $x(k)$ is defined by the estimation state $\widehat{x}(k)$ at $k$ th sampling instance.

We denote the general expression as follows:

$$
g(k, x(k))=\left[\begin{array}{c}
g_{11} x_{1}\left|x_{1}\right|+g_{12} x_{2}\left|x_{2}\right|+g_{13} x_{1} x_{2} \\
g_{21} x_{1}\left|x_{1}\right|+g_{22} x_{2}\left|x_{2}\right|+g_{23} x_{1} x_{2} \\
0 \\
0
\end{array}\right],
$$

where the nonlinear-term coefficient in (42) can be calculated by $M_{1}, T_{s}$, and $g_{c}(t, x(t))$ mentioned before.

Substitute (42) into (41), and we can get

$$
\bar{g}(k, x)=\left[\begin{array}{cccc}
\bar{g}_{1} & \bar{g}_{2} & 0 & 0 \\
\bar{g}_{3} & \bar{g}_{4} & 0 & 0 \\
0 & 0 & 0 & 0 \\
0 & 0 & 0 & 0
\end{array}\right],
$$

where

$$
\begin{aligned}
& \bar{g}_{1}=g_{11}\left|\widehat{x}_{1}\right|+g_{11} \hat{x}_{1} \operatorname{sgn}\left(\widehat{x}_{1}\right)+g_{13} \widehat{x}_{2}, \\
& \bar{g}_{2}=g_{12}\left|\widehat{x}_{2}\right|+g_{12} \hat{x}_{2} \operatorname{sgn}\left(\hat{x}_{2}\right)+g_{13} \widehat{x}_{1}, \\
& \bar{g}_{3}=g_{21}\left|\widehat{x}_{1}\right|+g_{21} \hat{x}_{1} \operatorname{sgn}\left(\widehat{x}_{1}\right)+g_{23} \hat{x}_{2}, \\
& \bar{g}_{4}=g_{22}\left|\hat{x}_{2}\right|+g_{22} \hat{x}_{2} \operatorname{sgn}\left(\hat{x}_{2}\right)+g_{23} \hat{x}_{1} .
\end{aligned}
$$

We assume that

$$
w(k)=w(k+1 \mid k)=\cdots=w\left(k+N_{p} \mid k\right),
$$

where $N_{p}$ is the predictive horizon. Then, considering that $w(k)$ is the difference between the estimation state $\widehat{x}(k)$ and $x(k)$ (calculated by $x(k-1), u(k-1)$, and $\bar{g}(k-1, x)$ ), this means

$$
\begin{aligned}
& w(k) \\
& =\hat{x}(k) \\
& \quad-[(A+\bar{g}(k-1, x)) x(k-1)+B u(k-1)] .
\end{aligned}
$$

Here the wave disturbance model can be considered as the state disturbance and we can use it to estimate the external disturbance in vertical plane of AUV motion.

Denote

$$
\bar{A}=A+\bar{g}(k-1, x)
$$

and then the iterative predictive states over $N_{p}$ at step $k$ is

$$
\begin{gathered}
\tilde{x}(k+1 \mid k)=\bar{A} \hat{x}(k)+B \widetilde{u}(k \mid k)+w(k), \\
\tilde{x}(k+2 \mid k)=\bar{A} \hat{x}(k+1)+B \widetilde{u}(k+1 \mid k)+w(k), \\
\vdots \\
\tilde{x}\left(k+N_{p} \mid k\right)=\bar{A} \hat{x}\left(k+N_{p}-1\right) \\
\quad+B \tilde{u}\left(k+N_{p}-1 \mid k\right)+w(k),
\end{gathered}
$$

where $\widetilde{u}\left(k+N_{p} \mid k\right)$ is the predictive input at step $k$.

When we implement MPC algorithm, an incremental predictive model is always required, and therefore the input $\widetilde{u}(k+i \mid k)$ can be replaced by $\Delta \widetilde{u}(k+i \mid k)$ which means

$$
\widetilde{u}(k+i \mid k)=\widetilde{u}(k+i-1)+\Delta \widetilde{u}(k+i \mid k)
$$

and here we assume that $\tilde{u}(k+i \mid k)$ will change just at every step $\left(i<N_{u}\right)$ and remain constant after step $k+i\left(N_{u} \leq i<\right.$ $\left.N_{p}-1\right)$.

With the assumption that the predictive value of $w(k)$ at sample instance $k$ is zero, the vector output prediction equation can be calculated and expressed in condensed form which predicts the future dynamic behavior of the AUV longitudinal motion over the horizon $N_{p}$ :

$$
\mathbf{y}=\Psi \hat{x}(k)+\Gamma u(k-1)+\Theta \mathbf{u},
$$

where

$$
\begin{aligned}
& \mathbf{y}=\left[\begin{array}{c}
\tilde{y}(k+1 \mid k) \\
\vdots \\
\tilde{y}\left(k+N_{p} \mid k\right)
\end{array}\right], \\
& \mathbf{u}=\left[\begin{array}{c}
\Delta \widetilde{u}(k \mid k) \\
\vdots \\
\Delta \widetilde{u}\left(k+N_{u}-1 \mid k\right)
\end{array}\right],
\end{aligned}
$$

$\Psi=\left[\begin{array}{c}C \bar{A} \\ C \bar{A}^{2} \\ \vdots \\ C \bar{A}^{N_{p}}\end{array}\right]$,

$$
\Gamma=\left[\begin{array}{c}
C B \\
C(\bar{A} B+B) \\
\vdots \\
\sum_{i=0}^{N_{p}-1} C \bar{A}^{i} B
\end{array}\right]
$$

$$
\boldsymbol{\Theta}=\left[\begin{array}{ccc}
C B & \cdots & 0 \\
C(\bar{A} B+B) & \cdots & 0 \\
\vdots & \ddots & \vdots \\
\sum_{i=0}^{N_{p}-1} C \bar{A}^{i} B & \cdots & \sum_{i=0}^{N_{p}-N_{u}} C \bar{A}^{i} B
\end{array}\right] .
$$


4.2. Consideration of Constraints. In order to guarantee the correct operation of the AUV, the constraints of the input elevator deflection $\delta \in R^{N_{u}}$ over control horizon $N_{u}$ and system output $\mathbf{y} \in R^{2 N_{p}}$ over predictive horizon $N_{p}$ are used to define $M$ and $\gamma$ for each time step $k$ considering the physical limitations of the driving device in practical implementation, and one obtains

$$
\begin{gathered}
\boldsymbol{\delta}_{\min } \leq \boldsymbol{\delta} \leq \boldsymbol{\delta}_{\max }, \\
\Delta \boldsymbol{\delta}_{\min } \leq \Delta \boldsymbol{\delta} \leq \Delta \boldsymbol{\delta}_{\max }, \\
\mathbf{y}_{\min } \leq \mathbf{y} \leq \mathbf{y}_{\max } .
\end{gathered}
$$

For simplification of the following discussion, and without loss of generality, we set the control horizon $N_{u}=2$, and then from (52) we can get

$$
\left[\begin{array}{c}
M_{1} \\
M_{2} \\
M_{3}
\end{array}\right] \Delta \boldsymbol{\delta} \leq\left[\begin{array}{c}
N_{1} \\
N_{2} \\
N_{3}
\end{array}\right],
$$

where

$$
\begin{aligned}
& M_{1}=\left[\begin{array}{c}
-\Lambda_{2} \\
\Lambda_{2}
\end{array}\right] \text {, } \\
& N_{1}=\left[\begin{array}{c}
-\boldsymbol{\delta}_{\min }+\Lambda_{1} \delta(k-1) \\
\boldsymbol{\delta}_{\max }-\Lambda_{1} \delta(k-1)
\end{array}\right] \text {, } \\
& M_{2}=\left[\begin{array}{c}
-I_{2 \times 2} \\
I_{2 \times 2}
\end{array}\right] \text {, } \\
& N_{2}=\left[\begin{array}{c}
-\Delta \boldsymbol{\delta}_{\min } \\
\Delta \boldsymbol{\delta}_{\max }
\end{array}\right] \text {, } \\
& M_{3}=\left[\begin{array}{c}
-\Theta \\
\Theta
\end{array}\right] \text {, } \\
& N_{3}=\left[\begin{array}{c}
-\mathbf{y}_{\min }+\Psi \hat{x}(k)+\Gamma \delta(k-1) \\
\mathbf{y}_{\max }-\Psi \hat{x}(k)-\Gamma \delta(k-1)
\end{array}\right], \\
& \Delta \boldsymbol{\delta}=\left[\begin{array}{c}
\Delta \delta(k) \\
\Delta \delta(k+1)
\end{array}\right] \text {, } \\
& \Lambda_{1}=\left[\begin{array}{l}
1 \\
1
\end{array}\right] \text {, } \\
& \Lambda_{2}=\left[\begin{array}{ll}
1 & 0 \\
1 & 1
\end{array}\right] \text {. }
\end{aligned}
$$

We describe (53) by

$$
M \Delta \boldsymbol{\delta} \leq \gamma
$$

which is equivalent to the constraints in the next section, and the constraints of the elevator deflection angle $\boldsymbol{\delta}$ and pitch angle $\theta$, corresponding to the inputs and outputs of the MPC, are characterized by $M$ and $\gamma$.
4.3. Optimization with Constraints. Conventional MPC performance index can be written as

$$
J=\left(\mathbf{y}-\mathbf{y}_{\text {ref }}\right)^{T} Q\left(\mathbf{y}-\mathbf{y}_{\text {ref }}\right)+\mathbf{u}^{T} R \mathbf{u},
$$

where $\mathbf{y}_{\text {ref }}$ is a future reference vector and $R$ and $Q$ are positive definite weighted matrices.

To simplify the expression, (56) can be rewritten as follows:

$$
\begin{aligned}
& \quad J=\frac{1}{2} \mathbf{u}^{T} E \mathbf{u}+\mathbf{u}^{T} F+\mathbf{f}_{0}, \\
& \text { s.t. } M \mathbf{u} \leq \gamma,
\end{aligned}
$$

where

$$
\begin{aligned}
E & =2\left(\boldsymbol{\Theta}^{T} \mathrm{Q} \boldsymbol{\Theta}+R\right) \\
F & =2 \boldsymbol{\Theta}^{T} \mathrm{Q}\left(\boldsymbol{\Psi} \widehat{x}(k)+\Gamma u(k-1)-\mathbf{y}_{\mathrm{ref}}\right), \\
\mathbf{f}_{0} & =\left(\boldsymbol{\Psi} \hat{x}(k)+\Gamma u(k-1)-\mathbf{y}_{\mathrm{ref}}\right)^{T} \\
& \cdot\left(\boldsymbol{\Psi} \hat{x}(k)+\Gamma u(k-1)-\mathbf{y}_{\mathrm{ref}}\right) .
\end{aligned}
$$

To minimize the quadratic function subject to (55), a QP (Quadratic Programming) problem has come out. Let us consider the expression which contains the Lagrange multipliers, that is, a QP problem subject to equality constraints $M \mathbf{u}=\gamma$, by the formula below:

$$
J=\frac{1}{2} \mathbf{u}^{T} E \mathbf{u}+\mathbf{u}^{T} F+\mathbf{f}_{0}+\lambda^{T}(M \mathbf{u}-\gamma) .
$$

The minimization of $J$ is to take the first partial derivatives with respect to $\mathbf{u}$ and $\lambda$, and we make them equal to zero and obtain the formula below:

$$
\begin{aligned}
& \frac{\partial J}{\partial \mathbf{u}}=E \mathbf{u}+M^{T} \lambda+F=0 \\
& \frac{\partial J}{\partial \lambda}=M \mathbf{u}-\gamma=0 .
\end{aligned}
$$

The minimization of $J$ can be made by finding the optimal $\mathbf{u}$ and $\lambda$ via (60) and (61) where

$$
\begin{aligned}
& \lambda=-\left(M E^{-1} M^{T}\right)^{-1}\left(\gamma+M E^{-1} F\right), \\
& \mathbf{u}=-E^{-1}\left(M^{T} \lambda+F\right)=\boldsymbol{\eta}-E^{-1} M^{T} \lambda,
\end{aligned}
$$

where $\boldsymbol{\eta}=-E^{-1} F$ is the global optimal solution.

The inequality constraints may comprise active constraints and inactive constraints in (55). We use both $M_{i}$ and $\gamma_{i}$ to form the $i$ th inequality constraint. If $M_{i} \mathbf{u}=\gamma_{i}$, an inequality constraint $M_{i} \mathbf{u} \leq \gamma_{i}$ can be considered as active, and if $M_{i} \mathbf{u}<\gamma_{i}$, it is inactive. Here we use the Kuhn-Tucker conditions [37] to define the active and inactive constraints in terms of $\lambda$. If the active set were known, the original problem could become equality constrains problem in (59).

In the conventional active set method [38], which belongs to the primal methods, the solutions are based on $\mathbf{u}$ (called 
decision variables). If the MIMO system has too many constraints, the calculations are complex and it is not a straightforward work.

A dual method can be used to identify the constraints which are inactive systematically. The inactive constraints can be eliminated in the solution, and $\lambda$ are called dual variables here. For constrained minimization problem, this method is a very simple programming procedure. The dual problem is derived from original primal problem as follows. Substituting (63) in (59), the dual problem is written as

$$
\min _{\lambda \geq 0}\left(\frac{1}{2} \lambda^{T} H \lambda+\lambda^{T} K+\frac{1}{2} \gamma^{T} E^{-1} \gamma\right),
$$

where the matrices $H$ and $K$ are given by

$$
\begin{aligned}
& H=M E^{-1} M^{T}, \\
& K=\gamma+M E^{-1} F .
\end{aligned}
$$

Subject to $\lambda \geq 0$, we minimize the dual performance index

$$
J=\frac{1}{2} \lambda^{T} H \lambda+\lambda^{T} K+\frac{1}{2} \gamma^{T} E^{-1} \gamma
$$

where the set of $\lambda$ are denoted as $\lambda^{*}$. By using Hildreth's QP procedure [39] the dual problem is solved and the method can be written as

$$
\lambda_{i}(k+1)=\max \left[0, \beta_{i}(k+1)\right]
$$

with

$$
\begin{aligned}
& \beta_{i}(k+1) \\
& =-\frac{1}{h_{i i}}\left[k_{i}+\sum_{j=1}^{i-1} h_{i j} \lambda_{j}(k+1)+\sum_{j=i+1}^{N_{k}} h_{i j} \lambda_{j}(k)\right],
\end{aligned}
$$

where $h_{i j}$ is the $i j$ th element in $H, k_{i}$ is the $i$ th element in $K$, and $N_{k}$ is the number of rows of $K$. In this method, there are $\lambda_{j}(k+1)$ and $\lambda_{j}(k)$ in one iterative cycle. And we set $\lambda_{j}(0)=0$, at $k=1$, and the iterative procedure will converge to $\lambda^{*}$ as a result. Substitute $\lambda^{*}$ into (63) and we have

$$
\mathbf{u}=\boldsymbol{\eta}-E^{-1} M^{T} \lambda^{*}
$$

where $\lambda^{*}=\left[\beta_{1}(k+1), \beta_{2}(k+1), \ldots, \beta_{N_{k}}(k+1)\right]^{T}$.

According to the receding horizon control in MPC, the first elements (elevator deflection $\Delta \delta(k)$ ) in $\mathbf{u}$ are taken to construct $\Delta u(t)$.

Remark 8. Because Hildreth's QP is a search-based pointby-point algorithm, there is no matrix inversion calculation. However, if the number of the active constraints is more than the number of u's or the active constraints are linearly dependent, then $\lambda$ will not converge to $\lambda^{*}$ and the iteration will terminate at the largest value of the iterative counter. But the algorithm will not end because there is no matrix inversion calculation. In this case, finally, the algorithm will end in a near-optimal solution with the violation of constraints. This is the reason why we use Hildreth's QP here, for its good ability to automatically recover from a deterioration constrained process.

\subsection{Steps of NROO-Based MPC-NMOL with Constraints}

(a) Set values of $N_{P}, N_{u}$, and specify $Q, R$.

(b) Get the estimation of current state $\hat{x}(k)$, sample current depth $z(t)$, and pitch angle $\theta(t)$.

(c) Calculate matrix $\bar{A}$ by online-linearization at current operating point which is defined by $\widehat{x}(k)$, to get matrixes $\Psi, \Gamma$, and $\Theta$. Update constraints matrixes $M$ and $\gamma$ by using $\delta(t-1)$, and then $E, F$, and $\mathbf{f}_{0}$ can be calculated.

(d) Check if the global optimal solution $\eta$ satisfies the constraints. If so, make $\lambda^{*}$ equal to zero vector and go to (f). If not, go to (e).

(e) Calculate matrices $H$ and $K$, and then the dual variable $\lambda^{*}$ can be calculated from (61).

(f) Get $\Delta u(t)$ from optimal solution $\mathbf{u}$.

(g) Go to step (b).

\section{Simulation Results}

In this paper, simulations are presented to demonstrate the effectiveness of NROO-based MPC-NMOL. The method is used in a given depth control of REMUS AUV which is developed by MIT (Massachusetts Institute of Technology). The values of nonlinear model parameters are shown as follows:

$$
\begin{aligned}
& A=\left[\begin{array}{cccc}
0.9921 & 0.0063 & 0 & 0.0002 \\
0.0453 & 0.9961 & 0 & -0.0071 \\
0.01 & 0 & 1 & -0.0151 \\
0 & 0.01 & 0 & 1
\end{array}\right] \\
& B=\left[\begin{array}{c}
-0.0065 \\
-0.04 \\
0 \\
0
\end{array}\right] \\
& C=\left[\begin{array}{llll}
0 & 0 & 1 & 0 \\
0 & 0 & 0 & 1
\end{array}\right] \text {, } \\
& D=\left[\begin{array}{cc}
0.0002 & 0 \\
0 & 0.0012 \\
0.0002 & 0 \\
0 & 0.0012
\end{array}\right] \\
& g(k, x(k))=M^{-1}\left[\begin{array}{c}
-0.02 x_{1}\left|x_{1}\right|-0.0066 x_{2}\left|x_{2}\right| \\
0.0046 x_{1}\left|x_{1}\right|-0.2272 x_{2}\left|x_{2}\right| \\
0 \\
0
\end{array}\right] \text {. }
\end{aligned}
$$

Here the physical parameters of REMUS AUV, which can be found from [40], are shown in Table 1. 


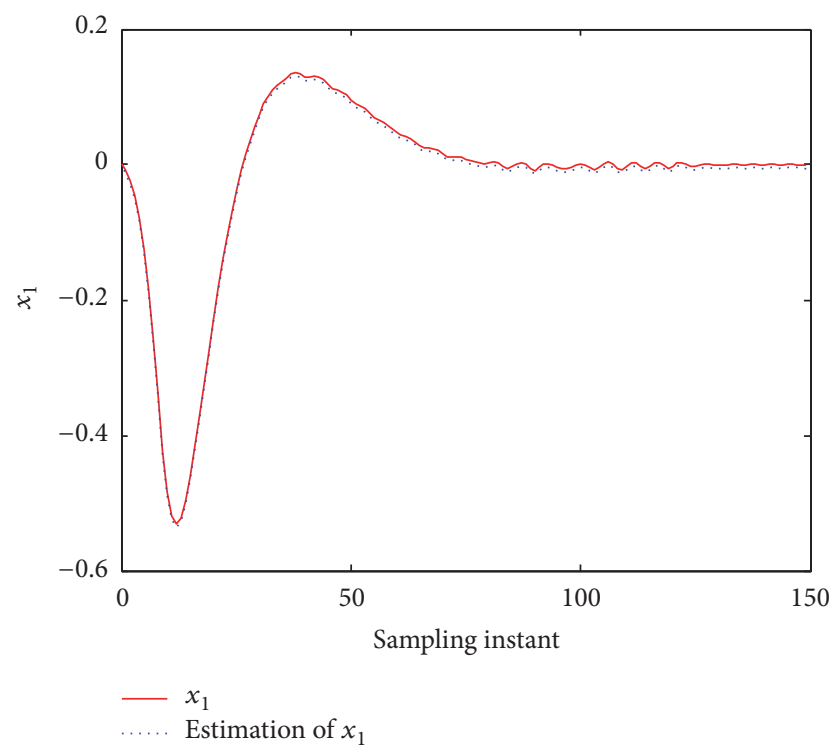

(a)

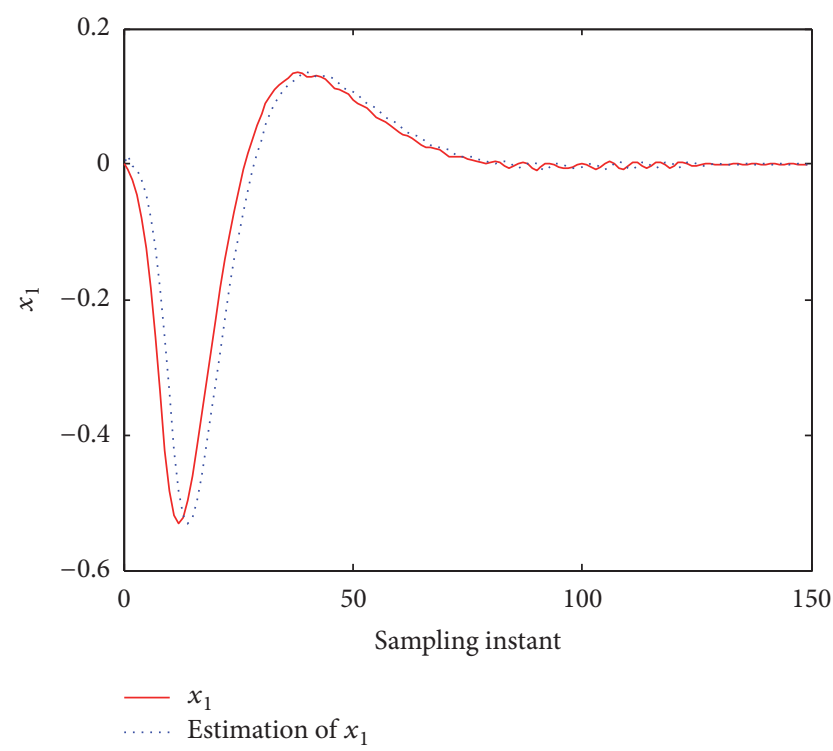

(b)

FIgURE 2: Comparison of $x_{1}$ and $\widehat{x}_{1}$ (a) using the NROO and (b) using the FOO.

5.1. Nonlinear Reduced-Order Observer Design. From the result that $\operatorname{rank}(C D)=\operatorname{rank}(D)=2$ and $(A, C)$ are observable, we can easily verify the existence of NROO.

By using Schmidt orthogonalization, we can get the matrix $T$ from the combination of $C_{1}$ and its standard orthogonal basis $C_{2}$. Obviously, the nonsingular matrix $T$ has only one form which is

$$
T=\left[\begin{array}{llll}
1 & 0 & 0 & 0 \\
0 & 1 & 0 & 0 \\
0 & 0 & 1 & 0 \\
0 & 0 & 0 & 1
\end{array}\right] .
$$

We choose $L_{g}=0.001$ as the value of the Lipschitz constant, and then the matrices parameter values in (19) can be calculated as follows:

$$
\begin{aligned}
& \bar{A}_{1}=\left[\begin{array}{ll}
0.9921 & 0.0063 \\
0.0453 & 0.9961
\end{array}\right], \\
& \bar{A}_{2}=\left[\begin{array}{cc}
0.01 & 0 \\
0 & 0.01
\end{array}\right] \text {, } \\
& C_{1}=\left[\begin{array}{cccc}
-1 & 0 & 0 & 0 \\
0 & -1 & 0 & 0
\end{array}\right], \\
& C_{2}=\left[\begin{array}{cccc}
0 & 0 & -1 & 0 \\
0 & 0 & 0 & -1
\end{array}\right] \text {, } \\
& D_{1}=\left[\begin{array}{cc}
0.0002 & 0 \\
0 & 0.0012
\end{array}\right] \text {, } \\
& D_{2}=\left[\begin{array}{cc}
0.0002 & 0 \\
0 & 0.0012
\end{array}\right] \text {. }
\end{aligned}
$$

TAble 1: Physical Parameters of REMUS AUV.

\begin{tabular}{lcc}
\hline Description & Values & Units \\
\hline$m$ & 30.48 & $\mathrm{~kg}$ \\
Length & 1.33 & $\mathrm{~m}$ \\
Beam & 0.26 & $\mathrm{~m}$ \\
$I_{x x}$ & 0.177 & $\mathrm{Kg} \cdot \mathrm{m}^{2}$ \\
$I_{y y}$ & 3.45 & $\mathrm{Kg} \cdot \mathrm{m}^{2}$ \\
$I_{z z}$ & 3.45 & $\mathrm{Kg} \cdot \mathrm{m}^{2}$ \\
Location of CG & $(0,0,0.0196)$ & $\mathrm{m}$ \\
Location of BG & $(-0.611,0,0)$ & $\mathrm{m}$ \\
\hline
\end{tabular}

With the help of MATLAB LMI toolbox, condition (20) is solved to obtain $\gamma_{1}=2.3561 \times 10^{3}$; at the same time, other results are found as follows:

$$
\begin{aligned}
\bar{P} & =\left[\begin{array}{cc}
144.6253 & -54.7977 \\
-54.7977 & 33.5797
\end{array}\right], \\
\bar{Y} & =\left[\begin{array}{cc}
42.6923 & -1.2754 \\
0.4957 & 41.8309
\end{array}\right], \\
\varepsilon_{1} & =4.3871 \times 10^{3} .
\end{aligned}
$$

One obtains

$$
\bar{G}=\bar{P}^{-1} \bar{Y}=\left[\begin{array}{ll}
0.7880 & 1.2135 \\
1.3007 & 3.2259
\end{array}\right] \text {. }
$$

Figures 2 and 3 show the estimation of $x_{1}(k)$ and $x_{2}(k)$ by using NROO and FOO. Although both observers can make the state estimation error converge asymptotically, compared to FOO, NROO has a better performance of state estimation. 


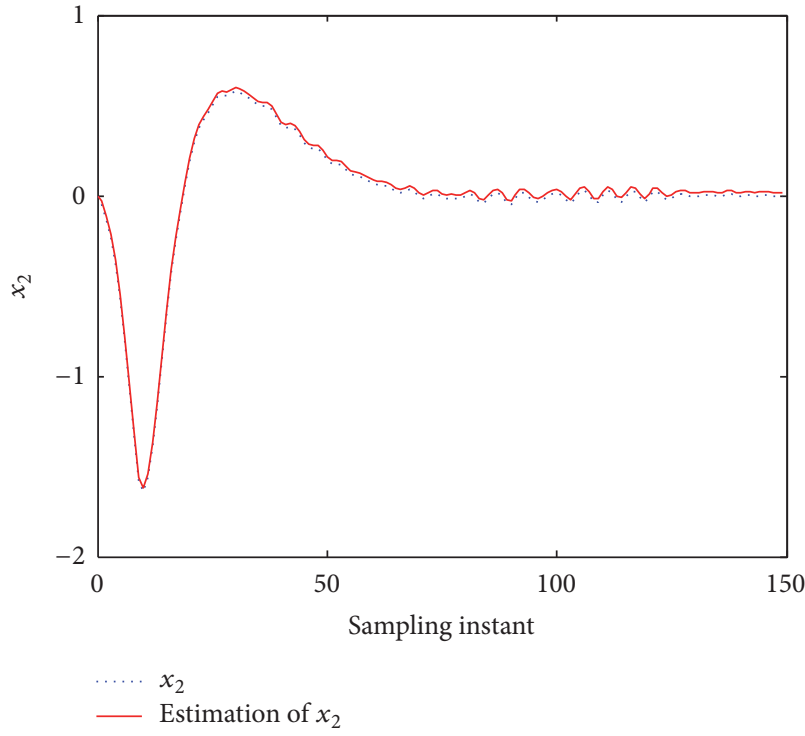

(a)

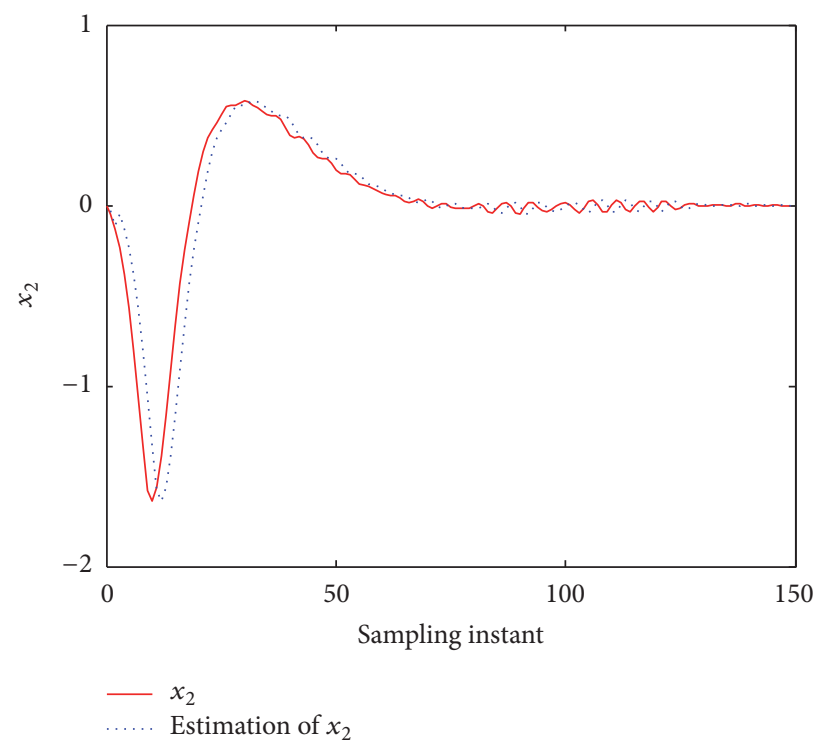

(b)

FIGURE 3: Comparison of $x_{2}$ and $\hat{x}_{2}$ (a) using the NROO and (b) using the FOO.

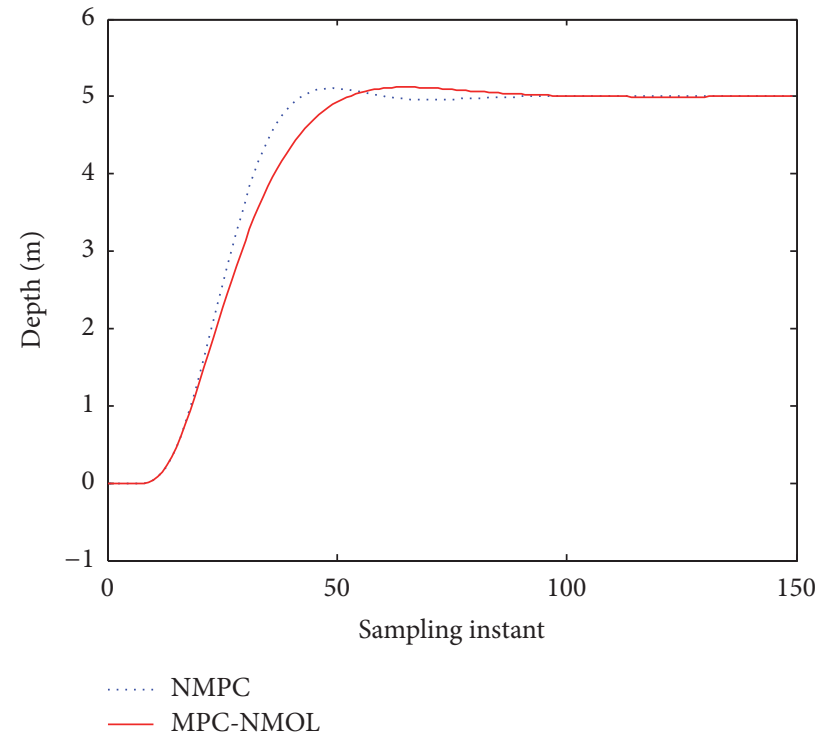

FIgURE 4: Comparison of depth with no disturbance.

5.2. Implementation of MPC-NMOL. The parameters of MPC are chosen as $N_{p}=30, N_{u}=2, Q=I_{N_{p} \times N_{p}}$, and $R=10$. The initial values of state variables are all zero. The waves disturbance exposed on REMUS is assumed at a level 3 sea state, $H_{s}=0.88(\mathrm{~m}), \beta=45^{\circ}, C_{d}=0.65, C_{m}=1.95$, and $N=$ 271. The surge speed is $U_{c}=1.51(\mathrm{~m} / \mathrm{s})$, the desired depth is $5(\mathrm{~m})$, and pitch angle is $0^{\circ}$. Input and output constraints are

$$
\begin{gathered}
\delta_{\max }=-\delta_{\min }=30^{\circ}, \\
\Delta \delta_{\max }=-\Delta \delta_{\min }=5^{\circ} / \mathrm{s}, \\
-90^{\circ} \leq \theta \leq 90^{\circ} .
\end{gathered}
$$

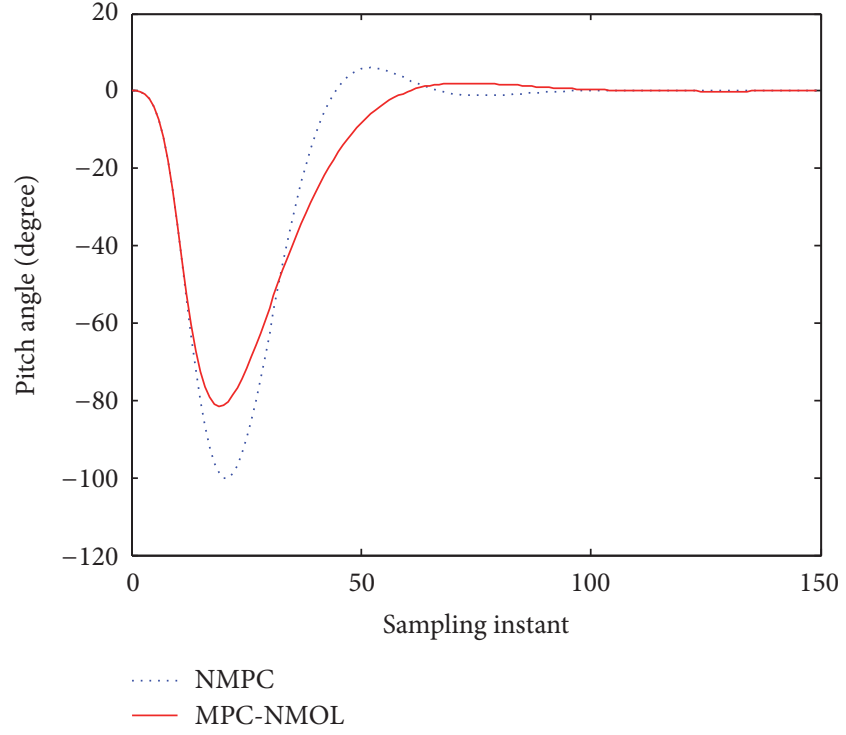

FIgURE 5: Comparison of pitch angle with no disturbance.

Case 1. First, we assume that there is no disturbance in simulation process, and NMPC is used to compare with the proposed method. Both of the two methods (MPC-NMOL and NMPC) have all state variables measurable. Figure 4 compares MPC-NMOL and NMPC simulation results of depth output. Figure 5 compares the simulation results of pitch output. Figure 6 compares the elevator deflection angle input.

Case 2. Next, it is assumed that wave disturbance affects the state process, and the other condition is the same as Case 1. Figures 7-9 show the comparison of depths, pitch angles, and elevator deflection angles with wave disturbance. 


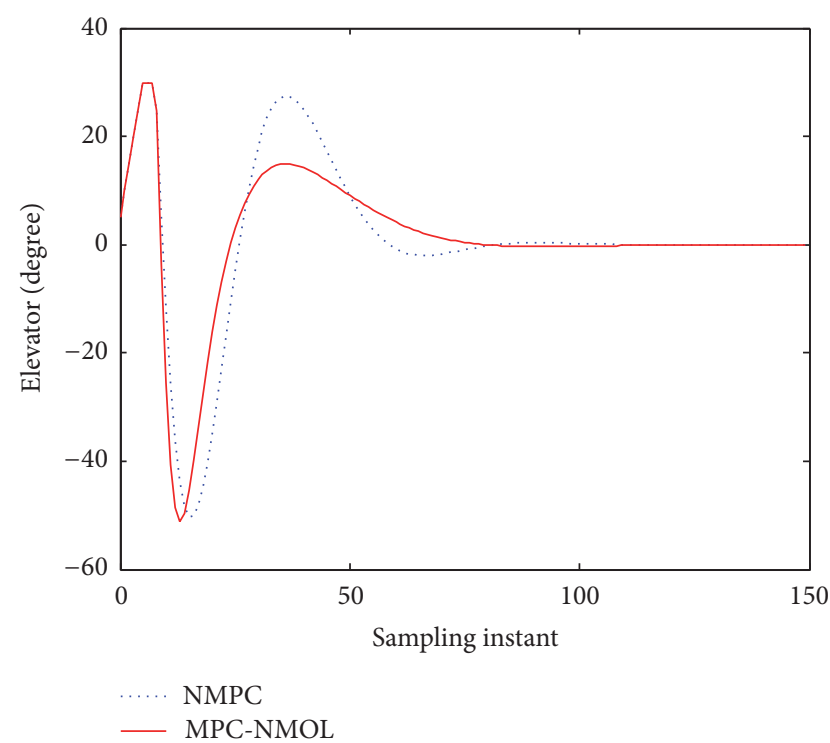

FIGURE 6: Comparison of elevator deflection angle with no disturbance.

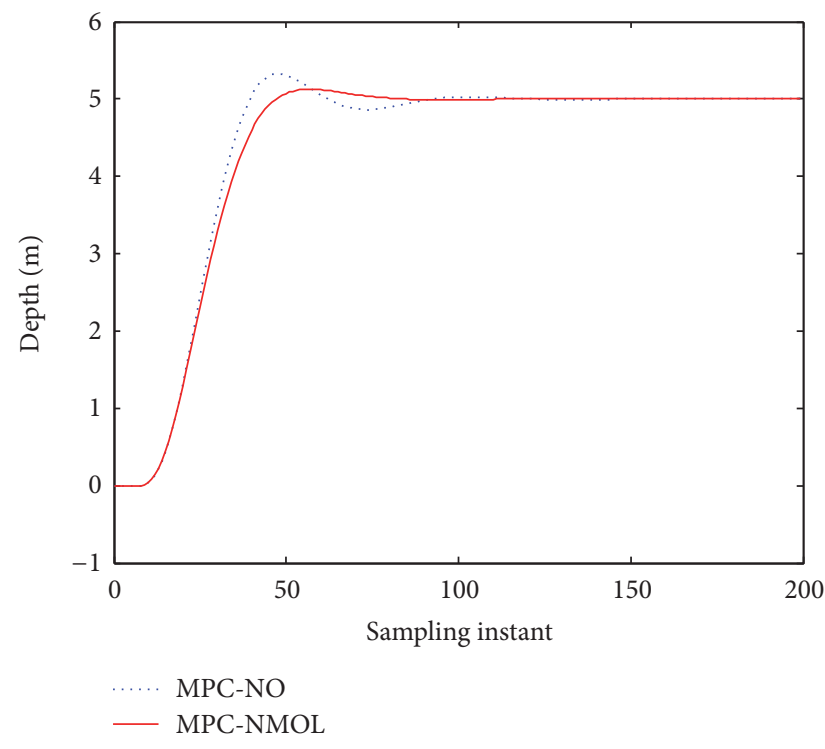

FIGURE 7: Comparison of depth with disturbance.

Figure 10 shows the wave force and moment, which can be seen as state process disturbance and assumed to be zeromean white noise sequence. The wave force and moment are calculated from (7), so they have the same form but different amplitude. Furthermore, when we simulate the wave force and moment, we choose multiple influential frequencies which are near the given main frequency of $P-M$ spectrum to superimpose the irregular waves.

All these results in Cases 1 and 2 demonstrate that AUV could achieve the desired depth and pitch angle under the wave disturbance. In addition, the input signals in MPCNMOL are smooth and without control signal saturation.

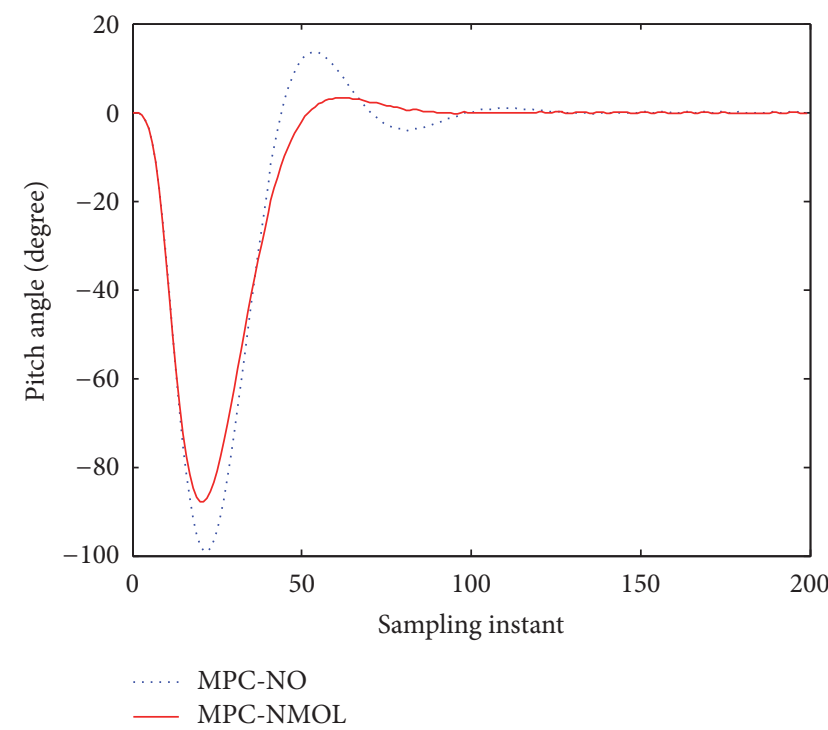

FIGURE 8: Comparison of pitch angle with disturbance.

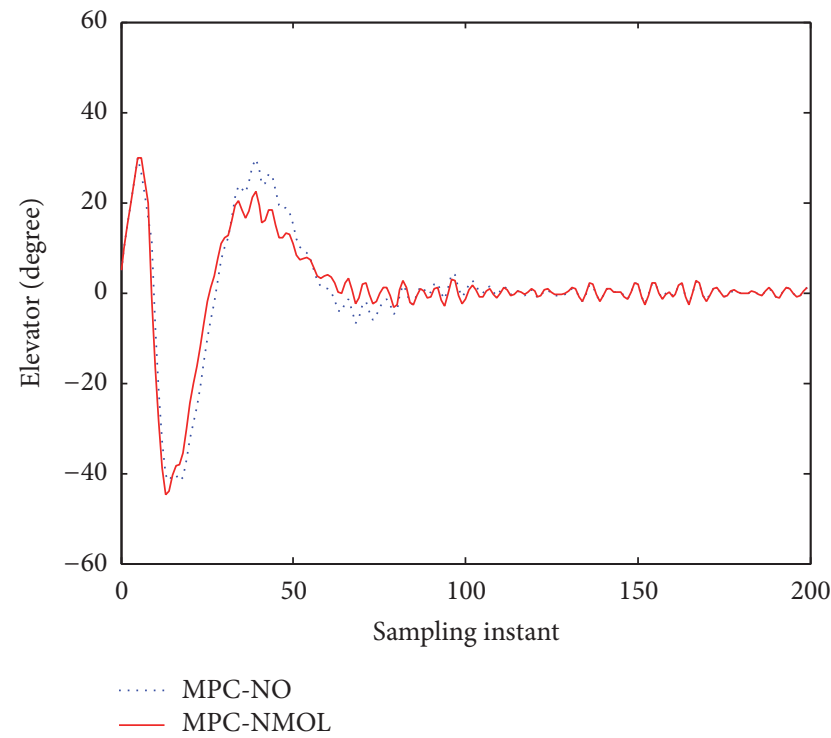

FIgURE 9: Comparison of elevator deflection angle with disturbance.

\section{Conclusions}

In this paper, a NROO-based model predictive controller with nonlinear model online linearization for AUV in vertical plane is presented, which controls the depth and pitch angle. This design uses the NROO to estimate the states used in MPC. The design process of the controller also takes into account the practical elevator deflection constraints and output constraints. By using a Hildreth's QP procedure, the constraints can be simply handled. Making use of the proposed MPC methods, the AUV can navigate in vertical plane with desired depth and pitch angle. It is robust against rough wave disturbance near surface. The simulations carried out provide the validation of the proposed methods, presenting 

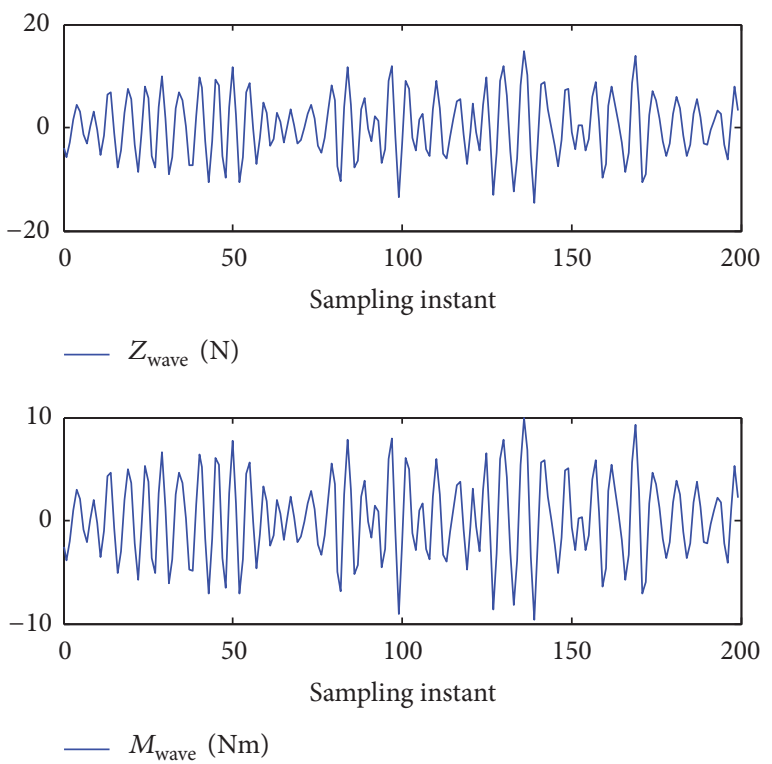

FIGURE 10: Wave force and moment.

fast dynamical response and strong robustness to external disturbances. Accurate control and state estimation can also be achieved.

\section{Competing Interests}

The authors declare that there is no conflict of interests regarding the publication of this paper.

\section{Acknowledgments}

The project is financially supported by the NNSF (National Natural Science Foundation) of China under Grant 51279039.

\section{References}

[1] X. Xiang, L. Lapierre, and B. Jouvencel, "Smooth transition of AUV motion control: from fully-actuated to under-actuated configuration," Robotics \& Autonomous Systems, vol. 67, pp. 1422, 2015.

[2] W. Chen, Y. Wei, J. Zeng, H. Han, and X. Jia, "Adaptive terminal sliding mode NDO-based control of underactuated AUV in vertical plane," Discrete Dynamics in Nature and Society, vol. 2016, Article ID 6590517, 9 pages, 2016.

[3] Z. Yan, H. Yu, and S. Hou, "Diving control of underactuated unmanned undersea vehicle using integral-fast terminal sliding mode control," Journal of Central South University, vol. 23, no. 5, pp. 1085-1094, 2016.

[4] G. V. Lakhekar, L. M. Waghmare, and P. S. Londhe, "Enhanced dynamic fuzzy sliding mode controller for autonomous underwater vehicles," in Proceedings of the IEEE Underwater Technology (UT '15), IEEE, Chennai, India, February 2015.

[5] A. Adhami-Mirhosseini, M. J. Yazdanpanah, and A. P. Aguiar, "Automatic bottom-following for underwater robotic vehicles," Automatica, vol. 50, no. 8, pp. 2155-2162, 2014.
[6] M. B. Loc, H.-S. Choi, S.-S. You, and T. N. Huy, "Time optimal trajectory design for unmanned underwater vehicle," Ocean Engineering, vol. 89, pp. 69-81, 2014.

[7] Y.-H. Tseng, C.-C. Chen, C.-H. Lin, and Y.-S. Hwang, "Tracking controller design for diving behavior of an unmanned underwater vehicle," Mathematical Problems in Engineering, vol. 2013, Article ID 504541, 10 pages, 2013.

[8] S.-P. Hsu and T.-S. Liu, "Modifications of control loop to improve the depth response of autonomous underwater vehicles," Mathematical Problems in Engineering, vol. 2014, Article ID 324813, 12 pages, 2014.

[9] B. Subudhi, K. Mukherjee, and S. Ghosh, "A static output feedback control design for path following of autonomous underwater vehicle in vertical plane," Ocean Engineering, vol. 63, pp. 72-76, 2013.

[10] S. Heshmati-Alamdari, A. Eqtami, G. C. Karras, D. V. Dimarogonas, and K. J. Kyriakopoulos, "A self-triggered visual servoing model predictive control scheme for under-actuated underwater robotic vehicles," in Proceedings of the IEEE International Conference on Robotics and Automation (ICRA '14), pp. 38263831, Hong Kong, China, June 2014.

[11] L. Medagoda and S. B. Williams, "Model predictive control of an autonomous underwater vehicle in an in situ estimated water current profile," in Proceedings of the (OCEANS '12) MTS/IEEE Yeosu Conference: The Living Ocean and Coast-Diversity of Resources and Sustainable Activities, Yeosu, Korea, May 2012.

[12] P. Jagtap, P. Raut, P. Kumar, A. Gupta, N. Singh, and F. Kazi, "Control of autonomous underwater vehicle using reduced order model predictive control in three dimensional space," IFAC-PapersOnLine, vol. 49, no. 1, pp. 772-777, 2016.

[13] C. V. Caldwell, D. D. Dunlap, and E. G. Collins Jr., "Motion planning for an autonomous underwater vehicle via sampling based model predictive control," in Proceedings of the MTS/IEEE Seattle (OCEANS '10), pp. 1-6, Seattle, Wash, USA, September 2010.

[14] D. C. Fernández and G. A. Hollinger, "Model predictive control for underwater robots in ocean waves," IEEE Robotics \& Automation Letters, vol. 2, no. 1, pp. 88-95, 2017.

[15] J. Gao, C. Liu, and A. Proctor, "Nonlinear model predictive dynamic positioning control of an underwater vehicle with an onboard USBL system," Journal of Marine Science \& Technology, vol. 21, no. 1, pp. 57-69, 2016.

[16] P. Hamelin, P. Bigras, J. Beaudry, P.-L. Richard, and M. Blain, "Discrete-time state feedback with velocity estimation using a dual observer: application to an underwater direct-drive grinding robot," IEEE/ASME Transactions on Mechatronics, vol. 17, no. 1, pp. 187-191, 2012.

[17] W. Zhang, Y. Guo, D. Meng, Z. Liang, and T. Chen, "Research on diving control of underactuated UUV based on model predictive control with artificial bee colony algorithm," in Proceedings of the 34th Chinese Control Conference (CCC '15), pp. 4073-4078, IEEE, Hangzhou, China, July 2015.

[18] Z. Gao, T. Breikin, and H. Wang, "Discrete-time proportional and integral observer and observer-based controller for systems with both unknown input and output disturbances," Optimal Control Applications \& Methods, vol. 29, no. 3, pp. 171-189, 2008.

[19] K. Zhang, B. Jiang, P. Shi, and A. Shumsky, "Reduced-order fault estimation observer design for discrete-time systems," in Proceedings of the 10th World Congress on Intelligent Control and Automation (WCICA '12), pp. 2959-2964, Beijing, China, July 2012. 
[20] J. C. Kinsey, Q. Yang, and J. C. Howland, "Nonlinear dynamic model-based state estimators for underwater navigation of remotely operated vehicles," IEEE Transactions on Control Systems Technology, vol. 22, pp. 1845-1854, 2014.

[21] S. Mahapatra, B. Subudhi, and R. Rout, "Diving control of an Autonomous Underwater Vehicle using nonlinear Ho measurement feedback technique," in Proceedings of the OCEANS 2016-Shanghai, April 2016.

[22] K. Zhang, B. Jiang, and P. Shi, "Fast fault estimation and accommodation for dynamical systems," IET Control Theory and Applications, vol. 3, no. 2, pp. 189-199, 2009.

[23] A. Mustafa, K. Munawar, F. M. Malik, M. B. Malik, M. Salman, and S. Amin, "Reduced order observer design with DMPC and LQR for system with backlash nonlinearity," Arabian Journal for Science \& Engineering, vol. 39, no. 8, pp. 6521-6530, 2014.

[24] K. Zhang, M. Staroswiecki, and B. Jiang, "Reduced-order observer-based fault estimation design for multiple inputmultiple output discrete-time systems," Proceedings of the Institution of Mechanical Engineers Part I Journal of Systems \& Control Engineering, vol. 226, no. 1, pp. 101-110, 2011.

[25] S. A. Davari, D. A. Khaburi, F. Wang, and R. M. Kennel, "Using full order and reduced order observers for robust sensorless predictive torque control of induction motors," IEEE Transactions on Power Electronics, vol. 27, no. 7, pp. 3424-3433, 2012.

[26] J. Rodas, R. Gregor, M. Rivera, Y. Takase, and M. Arzamendia, "Efficiency analysis of reduced-order observers applied to the predictive current control of asymmetrical dual three-phase induction machines," in Proceedings of the IEEE International Symposium on Sensorless Control for Electrical Drives and Predictive Control of Electrical Drives and Power Electronics (SLED/PRECEDE '13), pp. 1-7, München, Germany, October 2013.

[27] S. H. Saïd, N. B. Nasr, M. F. Mimouni, and F. MŚahli, "Output feedback predictive controller for a class of nonlinear systems," in Proceedings of the American Control Conference (ACC '10), July 2010.

[28] R. Gregor, J. Balsevich, and B. Bogado, "Reduced-order observer for rotor current estimation in speed control of dualthree phase induction machine," in Proceedings of the 3rd IEEE International Conference on Power Engineering, Energy and Electrical Drives, (PowerEng '11), pp. 1-6, Malaga, Spain, May 2011.

[29] V. Sundarapandian, "Reduced order observer design for nonlinear systems," Applied Mathematics Letters, vol. 19, no. 9, pp. 936-941, 2006.

[30] V. Sundarapandian, "Reduced order observer design for discrete-time nonlinear systems," Applied Mathematics Letters, vol. 19, no. 10, pp. 1013-1018, 2006.

[31] Z. Mao, B. Jiang, and P. Shi, "Fault-tolerant control for a class of nonlinear sampled-data systems via a Euler approximate observer," Automatica, vol. 46, no. 11, pp. 1852-1859, 2010.

[32] M. Calasan, N. Soc, V. Vujicic et al., "Review of marine current speed and power coefficien-mathematical models," in Proceedings of the 4th Mediterranean Conference on Embedded Computing (MECO '15), pp. 427-431, Budva, Montenegro, June 2015.

[33] J. P. J. Avila and J. C. Adamowski, "Experimental evaluation of the hydrodynamic coefficients of a ROV through Morison's equation," Ocean Engineering, vol. 38, no. 17-18, pp. 2162-2170, 2011.
[34] D. Nešić, A. R. Teel, and P. V. Kokotović, "Sufficient conditions for stabilization of sampled-data nonlinear systems via discretetime approximations," Systems and Control Letters, vol. 38, no. 4-5, pp. 259-270, 1999.

[35] K. Zhang, B. Jiang, and P. Shi, "Observer-based integrated robust fault estimation and accommodation design for discretetime systems," International Journal of Control, vol. 83, no. 6, pp. 1167-1181, 2010.

[36] W. Kratz, "Characterization of strong observability and construction of an observer," Linear Algebra \& Its Applications, vol. 221, pp. 31-40, 1995.

[37] D. P. Bertsekas, Nonlinear Programming, 1999.

[38] E. F. Camacho and C. B. Alba, Model Predictive Control, Springer Science \& Business Media, 2013.

[39] L. Wang, Model Predictive Control System Design and Implementation Using $M A T L A B^{\circledR}$, Springer Science \& Business Media, Berlin, Germany, 2009.

[40] T. Prestero, "Verification of a 6-degree of freedom simulation model for the REMUS AUV," 2001. 


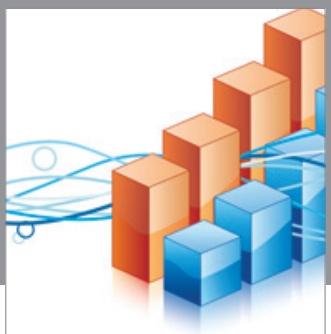

Advances in

Operations Research

vatem alat4

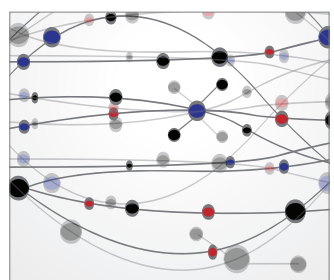

\section{The Scientific} World Journal
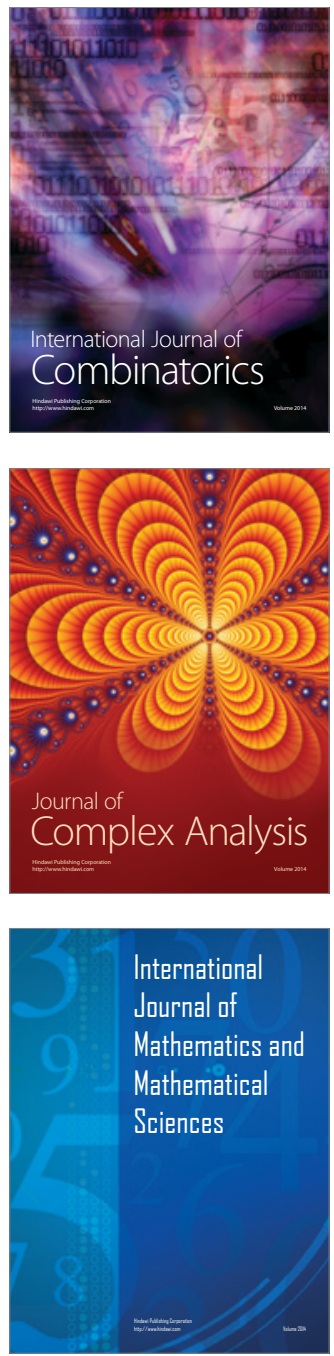
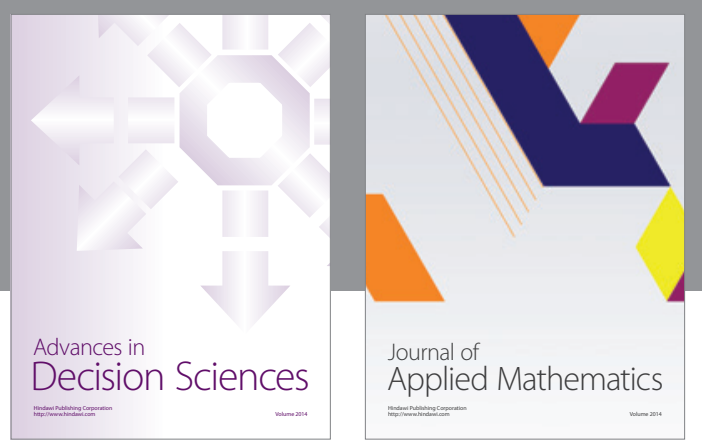

Algebra

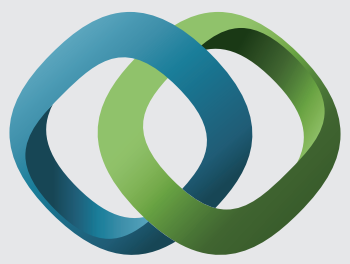

\section{Hindawi}

Submit your manuscripts at

https://www.hindawi.com
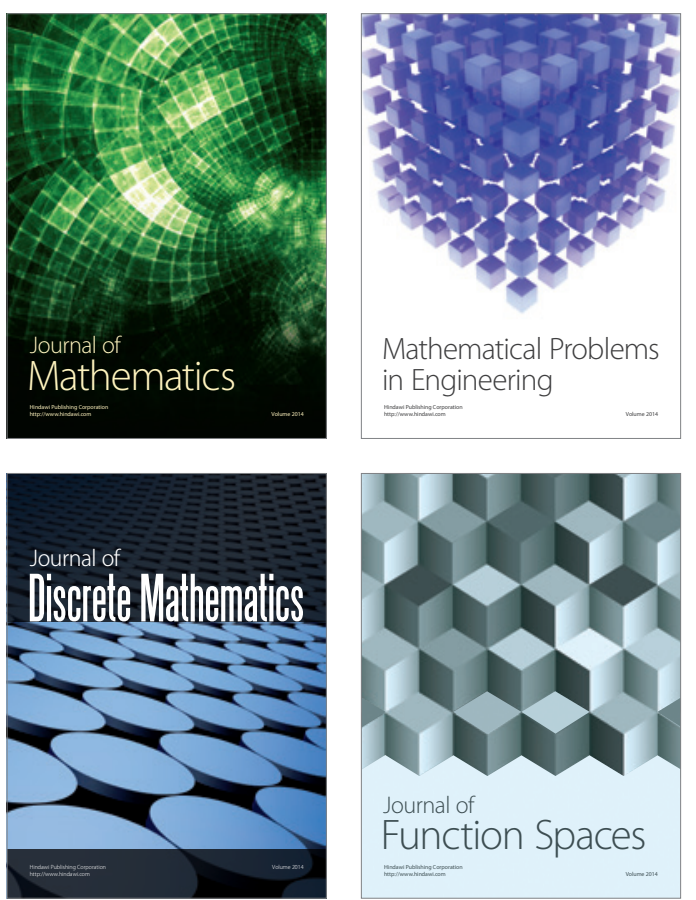

Mathematical Problems in Engineering
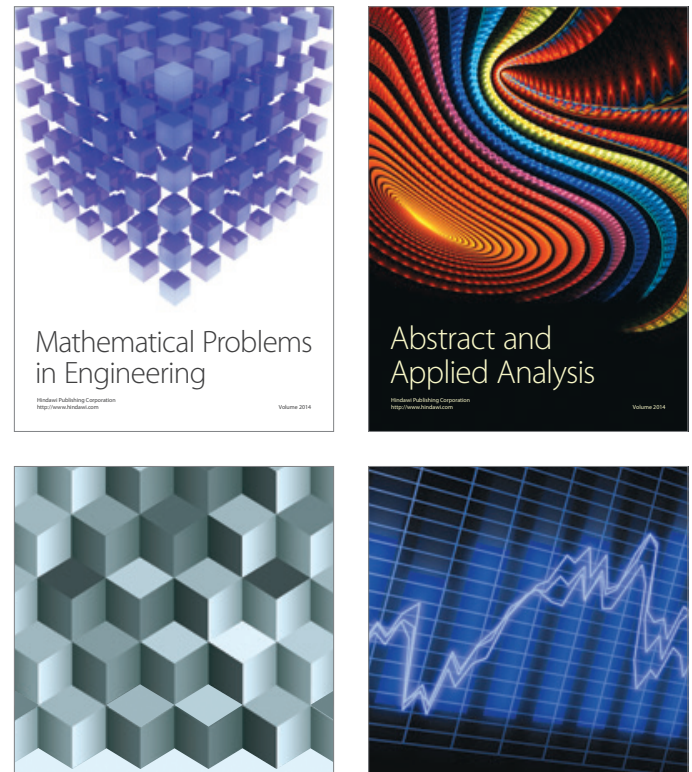

Journal of

Function Spaces

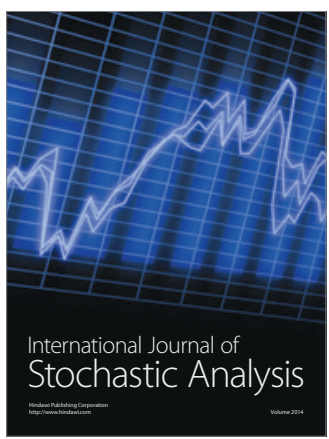

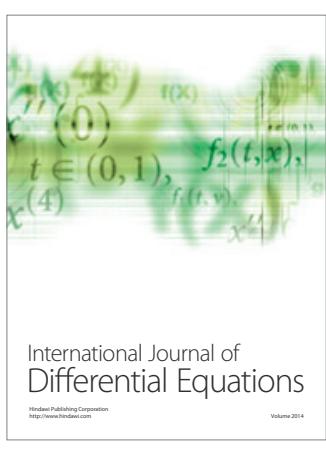
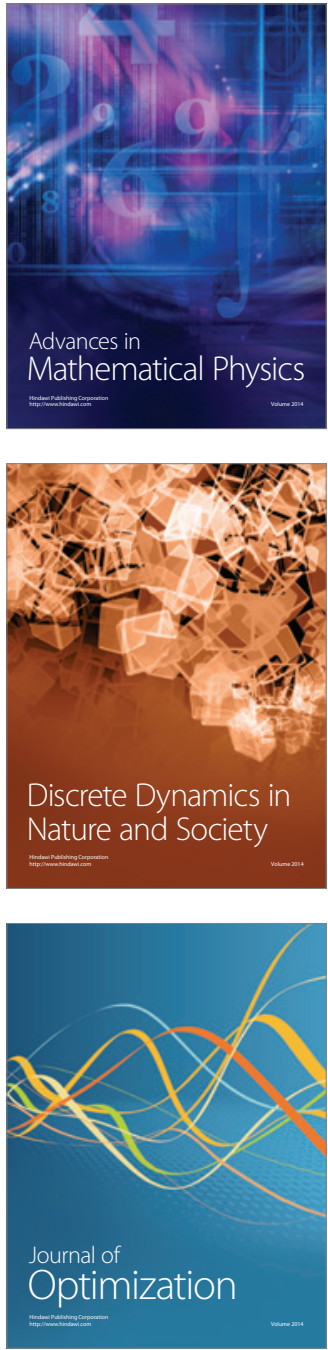